\title{
Solvability of the super KP equation and a generalization of the Birkhoff decomposition *
}

\author{
Motohico Mulase
}

Department of Mathematics, University of California, Los Angeles, CA 90024, USA

\begin{abstract}
Summary. The unique solvability of the initial value problem for the total hierarchy of the super Kadomtsev-Petviashvili system is established. To prove the existence we use a generalization of the Birkhoff decomposition which is obtained by replacing the loop variable and loop groups in the original setting by a super derivation operator and groups of infinite order super micro- (i.e. pseudo-) differential operators. To show the uniqueness we generalize the fact that every flat connection admits horizontal sections to the case of an infinite dimensional super algebra bundle defined over an infinite dimensional super space. The usual KP system with non-commutative coefficients is also studied. The KP system is obtained from the super KP system by reduction modulo odd variables. On the other hand, the first modified KP equation can be obtained from the super KP system by elimination of odd variables. Thus the super KP system is a natural unification of the KP system and the modified KP systems.
\end{abstract}

\section{Table of contents}



1. The KP system with non-commutative coefficients. . . . . . . . . . . . . . 6

2. The super KP system . . . . . . . . . . . . . . . . . . . . . . . . . . . . . . . . . . . . . . . . . . . . . . . 14

3. Generalized Birkhoff decomposition. . . . . . . . . . . . . . . . . . . . . . . . . 23

4. Some linear total differential equations . . . . . . . . . . . . . . . . . . . . . 31

5. Examples . . . . . . . . . . . . . . . . . . . . . . . . . . . . 42

References. . . . . . . . . . . . . . . . . . . . . . 46

\section{Introduction}

In this paper I will establish the complete integrability of the following new nonlinear partial differential equation

$$
f_{x t}-(3 / 4) f_{y y}+(3 / 2) f_{x x} f_{y}-(1 / 4) f_{x x x x}+(3 / 2) f_{x}^{2} f_{x x}+3 \lambda^{2} f_{x} f_{x x}=0
$$

\footnotetext{
* Research supported in part by NSF Grant No. DMS 86-03175
} 
where $f=f(x, y, t), \lambda$ is an arbitrary constant introduced in the process of integration and the subscripts denote partial derivatives. We introduce the following degrees of the variables: $\operatorname{deg} f=0, \operatorname{deg} \lambda=1, \operatorname{deg} x=-2, \operatorname{deg} y=-4$ and $\operatorname{deg} t=-6$. Then Eq. (1) is a homogeneous equation of degree 8. This equation is a kind of generalization of the Kadomtsev-Petviashvili equation

$$
u_{x t}-(3 / 4) u_{y y}-(1 / 4) u_{x x x x}-3 u_{x}^{2}-3 u u_{x x}=0 .
$$

Note that the linear terms of (1) and (2) are identical, but the nonlinear terms are quite different. (After the manuscript has been completed, Professor Ryogo Hirota kindly informed me that if one sets $\lambda=0$, then Eq. (1) reduces to a wellknown integrable equation called the First Modified KP equation, which admits bilinear expression.) Eq. (1) is a simplified version of the following system of two equations:

$$
\left\{\begin{array}{c}
f_{x t}-(3 / 4) f_{y y}+(3 / 2) f_{x x} f_{y}-(1 / 4) f_{x x x x}+(3 / 2) f_{x}^{2} f_{x x}+3 f_{x x} g=0 \\
f_{x x x} g_{x}-f_{x x} g_{x x}+f_{x x} g_{y}-f_{x y} g_{x}=0 .
\end{array}\right.
$$

If one substitutes $g$ of Eq. (3) by a solution $g=\lambda^{2} f_{x}$ of Eq. (4), then Eq. (3) reduces to Eq. (1).

What we shall do is to establish complete integrability of a certain system of infinitely many nonlinear partial differential equations and to show that Eq. (1) is nothing but the first closed differential equation among the system. The system we use is so-called the super KP system.

Let $x$ be an even variable and $\xi$ be an odd variable with $\xi^{2}=0$. We set $(R, \theta)=(\mathbb{C} \llbracket x, \xi \rrbracket, \partial / \partial \xi+\xi \partial / \partial x)$, which is an example of super-commuting super differential algebras. The Lax formalism for the super KP system is defined as follows:

$$
\left\{\begin{aligned}
\partial L / \partial t_{2 n} & =\left[L_{+}^{2 n}, L\right]=-\left[L_{-}^{2 n}, L\right] \\
\partial L / \partial t_{2 n-1} & =\left[L_{+}^{2 n-1}, L\right]-2 L^{2 n}+\sum_{k=1}^{\infty} t_{2 k-1}\left[L_{+}^{2 n+2 k-2}, L\right] \\
& =-\left[L_{-}^{2 n-1}, L\right]+\sum_{k=1}^{\infty} t_{2 k-1}\left(\partial L / \partial t_{2 n+2 k-2}\right)
\end{aligned}\right.
$$

for $n=1,2,3, \ldots$, where $[$,$] denotes the super commutator (defined in Sect. 2),$

$$
L=\theta+u_{1}+u_{2} \theta^{-1}+u_{3} \theta^{-2}+\ldots
$$

is a super micro-differential operator of order 1 , and $L_{+}^{n}$ (resp. $L_{-}^{n}$ ) denotes the terms of non-negative (resp. negative) powers of $\theta$. The even parameters $t_{2}, t_{4}, t_{6}, \ldots$ are usual commuting variables and the odd parameters $t_{1}, t_{3}, t_{5}, \ldots$ are Grassmann variables satisfying $t_{2 n-1} t_{2 m-1}=-t_{2 m-1} t_{2 n-1}$ and $\left(t_{2 n-1}\right)^{2}=0$. The first set (5) is identical with the Lax equation of the usual KP system, while (6) is quite different. The difference is due to the consistency of equations. If we set $\partial L / \partial t_{2 n-1}=\left[L_{+}^{2 n-1}, L\right]$, then it is meaningless because it implies $\theta=0$. On the other hand, if we set $\partial L / \partial t_{2 n-1}=-\left[L_{-}^{2 n-1}, L\right]$, then we get a meaningful 
equation for an individual $t_{2 n-1}$, but as a system it is not integrable. The expression (6) is the simplest equation which is completely integrable and involves the term $\left[L_{ \pm}^{2 n-1}, L\right]$ in it.

The importance of the usual KP system lies in the fact that it describes a universal family of isospectral deformations for an arbitrary ordinary differential operator. The form of Eq. (2) looks very special, but this equation and its total hierarchy of the KP system have a universality mentioned above. Moreover, it was this universality that led me to the discovery of the fact that every solution of the KP system naturally gives rise to a jacobian variety of an algebraic curve $[7,8]$. This fact was the essential part of my solution of the Schottky problem.

Because of its importance, various generalizations of the KP system have been proposed $[3,6,13]$. Among them, Manin and Radul [6] introduced the super KP system in the form of (5) and (6). In their paper, they assumed the solvability of a system of linear super differential equations (which is equivalent to our Eq. (9)) and the decomposability of its solution.

What we are going to do in this paper is to prove their assumptions. Moreover, we will show the following: If the operator $L$ of (7) satisfies the consistency condition

$$
\theta\left(u_{1}\right)+2 u_{2}=0
$$

which follows also from (5) and (6), then the initial value problem for the super $\mathrm{KP}$ system is uniquely solvable.

We will show that every solution $L$ of (5) and (6) gives rise to a solution $U$ of the universal equation

$$
d U=\Omega U,
$$

where $\Omega$ is the following formal 1 -form defined on an infinite dimensional super space;

$$
\Omega=\sum_{n=1}^{\infty} d t_{n} \theta^{n}+\sum_{n=1}^{\infty} \sum_{k=1}^{\infty} d t_{2 n-1} \cdot t_{2 k-1} \theta^{2 n+2 k-2}
$$

Conversely, every solution of (9) determines a solution $L$ of the super KP system uniquely. Therefore, the system of Eqs. (5) and (6) is equivalent to the linear total differential equation (9). Note that $\Omega$ satisfies the integrability condition $d \Omega=\Omega \wedge \Omega$. Therefore, the algebraic situation is the same as the usual KP system studied in [9]. From this point of view, we call the super KP system completely integrable in the sense of Frobenius.

Then how can we solve Eq. (9)? It is quite easy. Simply put

$$
U=\exp \left(\sum_{n=1}^{\infty} t_{n} \theta^{n}\right) \cdot U(0)
$$

where $U(0)$ is the initial value of $U$. Then it gives the unique solution of (9) with the initial value $U(0)$. Thus the operator $H=\exp \left(\sum_{n=1}^{\infty} t_{n} \theta^{n}\right)$ describes the 
time evolution of the super KP system. Note that $\Omega=d H \cdot H^{-1}$. Since the time evolution of the usual KP system is given by the operator $\exp \left(\sum_{n=1}^{\infty} t_{n}(d / d x)^{n}\right)$, the operator $H$ is a natural supersymmetric extension of the usual one. This is the very point of our motivation of introducing the super KP system.

Thus we have obtained a solution $U$ of Eq. (9). Then how can we find a solution $L$ of Eqs. (5) and (6)? This question is answered by a generalization of the Birkhoff decomposition. The original Birkhoff decomposition gives a factorization of a loop group into a product of subgroups consisting of loops which are boundary values of holomorphic functions defined on the upper hemisphere of $\mathbb{C P}^{1}$ containing $\infty$ and the lower hemisphere of $\mathbb{C P}^{1}$ containing 0 , together with a diagonal factor in between them, where we have identified the loop with the equator of $\mathbb{C P}^{1}$ (Pressley-Segal [10]). It also gives the famous theorem of Grothendieck: Every holomorphic vector bundle over $\mathbb{C P}^{1}$ is isomorphic to a direct sum of line bundles. The way we want to generalize this theorem is to replace the loop variable by a super derivation operator $\theta$. Thus the loop groups in the original setting will be replaced by groups of infinite order super micro-differential operators. Then the generalized Birkhoff decomposition gives us a way to construct a solution $L$ of the super KP system from a solution $U$ of (9). Actually, $L$ is given by $L=S \theta S^{-1}$, where $S$ is the "upper hemisphere part" of $U$.

A completely integrable system is always related with some kinds of group factorization. For example, the Toda lattice equations can be solved by the Bruhat decomposition, various two dimensional equations of classical and quantum models are solved by the original Birkhoff decomposition or the RiemannHilbert factorization, and the soliton equations by the generalized Birkhoff decomposition of infinite order micro-differential operators. What I am going to present in this paper is that if we go further in this direction toward a supersymmetric generalization of the group factorization, then we will encounter the super KP system in a very natural way. From this factorization point of view, the solution space of integrable systems always look like

\section{some "general" linear group/maximal "parabolic" subgroup.}

The appearance of the Grassmann varieties in the studies of integrable systems can be understood also from this point of view.

I would like to emphasize that the super KP system is not a trivial formalism generalization of the KP system. Indeed, Eq. (1) cannot be obtained from the framework of KP equations. Of course, all the usual KP equations can be recovered from the super KP system by reduction modulo odd variables. On the other hand, we can eliminate the odd evolution parameters from Eqs. (5) and (6) so that we obtain nonlinear partial differential equations in usual even variables alone. Eq. (1) is an example of such equations. The interesting fact is that the result of elimination does not agree with that of reduction. Actually, if we reduce Eq. (1) to the KP framework, then we get a trivial equation $0=0$ ! Therefore, a supersymmetric extension produces new equations.

This paper is organized as follows. In Sect. 1, we generalize the usual KP framework to the case of non-commutative coefficients. Recently, importance 
of non-commutative geometry has been emphasised by various people $[1,2,5,15]$. Our attempt was motivated by these trends. We will give a thorough treatment of the subject in this section because most of the techniques we need in the supersymmetric extension already appear in the theory with non-commutative coefficients. The main theorem in this section is the unique solvability of the Cauchy problem for the KP system with non-commuting coefficients (Theorem 1.4). Since our method of solving the equation is explicit, we can give a good convergence condition of the solution if the differential algebra has a norm (Remark 1.5). Also it will be shown that there is a unique solution even for an initial datum with arbitrary poles and logarithmic singularities. Therefore, in the framework of KP system of commutative coefficients, our solution space, which is an affine space, is larger than any proposed solution spaces. For example, it contains Sato's universal Grassmann manifold [11] and the loop space Grassmannian of Segal-Wilson [12]. Thus all the compact complex Grassmann varieties are embedded in our affine space (Remark 1.6).

In Sect. 2, we will introduce the super KP system motivated by the supersymmetric extension of $\exp \left(\sum_{n=1}^{\infty} t_{n} \partial^{n}\right)$ to $\exp \left(\sum_{n=1}^{\infty} t_{n} \theta^{n}\right)$. An almost perfect parallelism holds between the usual KP system and the super KP system. However, there is a big difference between them. In the super case, the 1 -form $\Omega$ of $(10)$ satisfies the zero-curvature condition $d \Omega=\Omega \wedge \Omega$, but $d \Omega \neq 0$. On the other hand, in the usual KP case, the corresponding 1 -form $\Omega$ satisfies $d \Omega=\Omega \wedge \Omega=0$. Therefore, from this fact, we can again realize that the super KP system is an essentially non-trivial generalization. The unique solvability of the initial value problem for the super KP system (Theorems 2.1 and 2.3) are the main theorems of this section.

In Section 3 we will first establish the generalized Birkhoff decomposition of a group of infinite order micro-differential operators. For a commutative case, the theorem was announced in [9]. Our Theorem 3.2 is the widest extension of the statement therein. Even though the groups are purely formal objects, we can establish actual convergence of the solution of the KP system because the group factorization gives an explicit formula of a solution depending on its initial data, as mentioned above. A supersymmetric analogue of the decomposition will also be proved, which was one of the assumptions of Manin-Rudul [6].

Section 4 is devoted to give the technical proofs of the statement we need in Sections 1 and 2 to establish the uniqueness for the (super) KP system. The theorem we need is the following. Let $\omega$ be a Lie algebra valued 1-form satisfying the zero-curvature condition $d \omega=(1 / 2)[\omega, \omega]$. Then there is a Lie group valued solution $Y$ of the linear total differential equation $d Y=\omega Y$. The statement is trivial if the Lie algebra and the manifold we are talking about are both of finite dimension. In our case, however, the algebra we use is the set of (super) differential operators and the base manifold is an infinite dimensional (super) space. Theerefore we need a careful treatment. We will use some interesting formulas in this section.

In the final section, we will compute actual shape of differential equations involved in the (super) KP system. We will derive Eq. (1) as the first non-trivial 
equation among the super KP system which is a single closed equation, namely, it has only finitely many terms and has a single unknown function. Some of the exact solutions of (1) will also be given. They correspond to rational, soliton and quasi-periodic solutions of usual soliton equations. These solutions are given by a quotient of two Jacobi elliptic functions and their degenerate functions. This gives another contrast between Eqs. (1) and (2), since the simplest solutions of the original KP equation are given naturally by the Weierstrass $\wp$-functions.

Once we establish the unique solvability of the initial value problem for the super KP system, we can define the super KP dynamical system $(X, T)$ as in [8], where $X$ is the set of all operators $L$ of the form (7) satisfying the consistency condition (8) and $T=\left\{\left(t_{1}, t_{2}, t_{3}, t_{4}, \ldots\right)\right\}$ is the group of super evolution variables. As the KP dynamical system has significant importance in algebraic geometry and many related areas, it can be expected that the super KP dynamical system will play an even more important role in the non-commutative geometry. These subjects will be treated in a separate article.

There are other approaches to supersymmetric extensions of the KP system (see literature cited in [6]). Recently, Ueno and Yamada [13] found a supersymmetric extension of Sato's work [11] on the KP system and gave a formula for the solutions in terms of infinite size super matrices. Their solutions coincide with ours if the super differential algebra is $\mathbb{C} \llbracket x, \xi \rrbracket$. They also obtained a super analogue of Hirota-Sato's $\tau$-functions. However, the $\tau$-function method does not fit for the purpose of establishing the solvability of the Cauchy problem.

During the manuscript of this paper was being typed, Professor Robert $M$. Miura kindly informed me of some of the explicit formulas of conservation laws for Eq. (1). The first two of them are the following:

$$
\begin{aligned}
\left(f_{x}\right)_{t}= & {\left[(1 / 4) f_{x x x}-(3 / 2) \lambda^{2} f_{x}^{2}-(1 / 2) f_{x}^{3}-(3 / 2) f_{x} f_{y}\right]_{x}+(3 / 4)\left[f_{x}^{2}+f_{y}\right]_{y}, } \\
\left(f_{x}^{2}\right)_{t}= & {\left[(1 / 2) f_{x} f_{x x x}-(1 / 4) f_{x x}^{2}-2 \lambda^{2} f_{x}^{3}-(3 / 4) f_{x}^{4}-(3 / 2) f_{x}^{2} f_{y}-(3 / 4) f_{y}^{2}\right]_{x} } \\
& +(1 / 2)\left[f_{x}^{3}+3 f_{x} f_{y}\right]_{y} .
\end{aligned}
$$

I do not know whether Eq. (1) or the system of Eqs. (3) and (4) is related to any model of 2-spatial 1-time dimensional classical fluid dynamics. However, since there are soliton-like solutions (see Sect. 5) and conservation laws for Eq. (1), it is natural to believe that there is such a model. On the other hand, the study of total hierarchy of the super KP equations and its solution space may have an importance in super string theories and moduli theories of super algebraic curves.

I would like to express my gratitude to Professor R.M. Miura and Professor R. Hirota for valuable comments.

\section{The KP system with non-commutative coefficients}

In this section we study a generalized KP system with non-commutative coefficients and show how the group decomposition theorem of Sect. 3 establishes its unique solvability for an arbitrary initial datum. 
Let $(R, \partial)$ be a differential algebra defined over a field $k$ of characteristic zero with the unity 1 . Namely, $R$ is an associative $k$-algebra with unity and $\partial: R \rightarrow R$ is a $k$-linear map satisfying the Leibnitz rule $\partial(f g)=\partial(f) \cdot g+f \partial(g)$ for all $f$ and $g$ in $R$. We do not assume commutativity of $R$.

One of the mathematical motivations of the KP system is to find a universal family of isospectral deformations of an arbitrary ordinary differential operator. The differential operators we will consider are elements of

$$
D=\left\{P=\sum_{n=0}^{\infty} a_{n} \partial^{n} \mid a_{n} \in R, a_{n}=0 \text { for large } n\right\} .
$$

Following Gelfand's idea, we use the set of formal ordinary micro-differential operators

$$
E=\left\{P=\sum_{v \in \mathbf{Z}} a_{v} \partial^{v} \mid a_{v} \in R, a_{v}=0 \text { for } v \gg 0\right\} .
$$

The set $E$ becomes an associative algebra by virtue of the general Leibnitz rule

$$
\partial^{v} \cdot f=\sum_{i=0}^{\infty}\left(\begin{array}{l}
v \\
i
\end{array}\right) f^{(i)} \partial^{v-i}
$$

for $v \in \mathbf{Z}$ and $f \in R$, where $f^{(i)}=\partial^{i}(f)$ and the binary coefficients $\left(\begin{array}{l}v \\ i\end{array}\right)$ are defined by using $\Gamma$-functions. An operator $P \in E$ is said to be normalized if its leading coefficient is one (i.e. monic) and the second top term is zero;

$$
P=\partial^{v}+0 \cdot \partial^{v-1}+\text { lower order terms. }
$$

Twenty years ago Lax [4] established the following. A parameter depending family $\{P(t)\}$ of ordinary differential operators is an isospectral family if and only if $P(t)$ satisfies the Lax equation

$$
\frac{d P(t)}{d t}=[Q(t), P(t)]
$$

with another parameter depending differential operator $Q(t)$, where $[A, B]$ $=A B-B A$. The reason why we have to use a differential operator $Q(t)$ is because we want an isospectral family of differential operators. If we use a micro-differential operator $Q(t) \in E$, then $\{P(t)\}$ will no longer be a family of differential operators, while the spectral structure is still preserved.

An operator $P=\sum_{v \in \mathbf{Z}} p_{v} \partial^{v} \in E$ is said to be of order $N$ if $p_{N} \neq 0$ and $p_{v}=0$ for all $v>N$. A normalized operator $P \in E$ of order $n>0$ has a unique normalized $n$-th root in $E$. Assume that $P(t) \in D$ is normalized of order $N$ and

$$
L(t)=P(t)^{1 / N}=\partial+u_{2} \partial^{-1}+u_{3} \partial^{-2}+\ldots \in E
$$


be its $N$ 'th root. It is easy to show that the Lax equation (1.4) is equivalent to

$$
\frac{d L(t)}{d t}=[Q(t), L(t)]
$$

Let $E^{(-1)}$ be the set of all micro-differential operators of order at most -1 . Then we have a natural left $R$-module direct sum decomposition

$$
E=E^{(-1)} \oplus D .
$$

According to (1.7), every $P \in E$ decomposes into $P=P_{-} \oplus P_{+}$, where $P_{-} \in E^{(-1)}$ and $P_{+} \in D$.

Since $L(t)$ has the form of (1.5), its $t$-derivative $d L(t) / d t$ is in $E^{(-1)}$. Therefore $Q(t)$ in (1.6) must satisfy

$$
[Q(t), L(t)] \in E^{(-1)}
$$

to make sense of (1.6). Therefore, to find out all possible isospectral deformations of $P=L^{N} \in D$, we have to determine all differential operators $Q \in D$ such that (1.8) holds. Note that $\left(L^{n}\right)_{+} \in D$ satisfies (1.8) because

$$
\left[\left(L^{n}\right)_{+}, L\right]=\left[L^{n}-\left(L^{n}\right)_{-}, L\right]=-\left[\left(L^{n}\right)_{-}, L\right] \in E^{(-1)} .
$$

Its converse is true if $(R, \partial)$ satisfies a special condition:

Lemma 1.1. Let $L \in E$ be a normalized first order operator. If $\operatorname{ker}(R \stackrel{\partial}{\rightarrow} R)$ is contained in the center of $R$, then the following conditions for $Q \in D$ are equivalent;

(a) $[Q, L] \in E^{(-1)}$.

(b) $Q$ is a linear combination of $\left(L^{n}\right)_{+}$'s over $\operatorname{ker} \partial$.

The proof can be easily performed by an induction on the order of $Q$. The above lemma tells us that essentially $\left(L^{n}\right)_{+}$'s give all possible isospectral deformations of $P=L^{N}$. It is natural to introduce distinct deformation parameters for different deformations. Thus we obtain the KP system

$$
\frac{\partial L}{\partial t_{n}}=\left[\left(L^{n}\right)_{+}, L\right]
$$

If $k=\operatorname{ker}(R \stackrel{\partial}{\rightarrow} R)$, then Eq. (1.9) gives a universal family of isospectral deformations of $P=L^{N}$. The advantage of using $L=P^{1 / N}$ instead of $P$ itself is that (1.9) does not refer to the order of $P$. Therefore (1.9) is a master equation of all universal families for any normalized differential operator. If we want to find a universal family of a differential operator $P$ of order $m$, then we solve (1.9) for $L=\partial+u_{2} \partial^{-1}+u_{3} \partial^{-2}+\ldots$ with an extra condition $L^{m}=P \in D$. For more detail, see [8].

To establish solvability of (1.9), we have to specify $t$-dependence of operators. So we define

$$
\mathscr{R}=R \llbracket t_{1}, t_{2}, t_{3}, \ldots \rrbracket
$$


as a projective limit algebra with a valuation

$$
\operatorname{val}_{t}: \mathscr{R} \backslash\{0\} \rightarrow \mathbf{N}=\{0,1,2,3, \ldots\}
$$

defined by $\operatorname{val}_{t}\left(t_{n}\right)=n, n=1,2,3, \ldots$ The $\mathscr{R}$-valued operators are defined by

$$
\mathscr{D}=\left\{P=\sum_{n=0}^{\infty} a_{n} \partial^{n} \mid a_{n} \in \mathscr{R}, a_{n}=0 \text { for } n \gg 0\right\}
$$

and

$$
\mathscr{E}=\left\{P=\sum_{v \in \mathbf{Z}} a_{v} \partial^{v} \mid a_{v} \in \mathscr{R}, a_{v}=0 \text { for } v \gg 0\right\}
$$

For a given normalized first order operator $L \in \mathscr{E}$, we define formal 1-forms by

$$
Z_{ \pm}^{L}= \pm \sum_{n=1}^{\infty} d t_{n}\left(L^{n}\right)_{ \pm}
$$

Then the Lax equation for the KP system is given by

$$
d L=\left[Z_{+}^{L}, L\right]=\left[Z_{-}^{L}, L\right]
$$

where $d=\sum_{n=1}^{\infty} d t_{n} \partial / \partial t_{n}$ denotes the exterior derivative in $t$.

To obtain an actual family of isospectral deformations, Eq. (1.14) must satisfy the integrability condition

$$
d Z_{ \pm}^{L}=Z_{ \pm}^{L} \wedge Z_{ \pm}^{L}
$$

Equation (1.15) is equivalent to the compatibility condition of deformations. Namely, (1.15) implies that the $t_{n}$-deformation followed by the $t_{m}$-deformation always coincides with the otherway around for all $m$ and $n$. One of the interesting facts of the KP system is that (1.15) follows automatically from (1.14). Therefore, a solution $L(t) \in \mathscr{E}$ of (1.14) always gives a family. However this does not itself imply that (1.14) is actually solvable. Indeed, the Cauchy problem of (1.14) for a general non-commutative differential algebra is not known.

To simplify Eq. (1.14) we introduce a group of inner automorphisms of $E$.

We define

$$
G=\left\{\sum_{n=0}^{\infty} s_{n} \partial^{-n} \mid s_{0}=1, s_{n} \in R\right\}
$$

and

$$
\mathscr{G}=\left\{\sum_{n=0}^{\infty} s_{n} \partial^{-n} \mid s_{0}=1, s_{n} \in \mathscr{R}\right\}
$$

Lemma 1.2. If $R \stackrel{\hat{a}}{\rightarrow} R$ is surjective (i.e. if indefinite integration is always possible in $R$ ), then for every normalized first order operator $L$ of $D$ (resp. $\mathscr{D})$, there is an invertible operator $S \in G$ (resp. $\mathscr{G})$ such that $S^{-1} L S=\partial$. 
The reason why we want to bring $L$ into $\partial$ is because $L=\partial$ satisfies the KP system (1.14) trivially. Our idea is to produce a solution of (1.14) by applying an inner automorphism $S$ to the simplest solution $\partial$.

Lemma 1.3. If $\partial: R \rightarrow R$ is surjective, then the Lax equation (1.14) is equivalent to the following system for $S \in \mathscr{G}$;

$$
\left\{\begin{array}{c}
L=S \partial S^{-1} \\
d S=Z_{-}^{L} \cdot S
\end{array}\right.
$$

We call the following equation

$$
d S=-\sum_{n=1}^{\infty} d t_{n}\left(S \partial^{n} S^{-1}\right)_{-} \cdot S
$$

the generalized KP system. If the differential algebra $(R, \partial)$ satisfies

$$
0 \rightarrow k \rightarrow R \stackrel{\partial}{\rightarrow} R \rightarrow 0 \text { exact, }
$$

then Eq. (1.19) is equivalent with the Lax equation of usual KP system and describes a universal family of isospectral deformations of normalized differential operators. For typical examples of such an algebra, we have $R=k \llbracket x \rrbracket$ and $\mathbb{C}((x))[\log x]$, the latter is the set of all polynomials in $\log x$ with coefficients in arbitrary Laurent series in $x$.

For more general $(R, \partial)$, Eq. (1.19) does not give a universal family of isospectral deformations. However, the reason why we introduced (1.19) is that the Cauchy problem of (1.19) is always solvable for any $(R, \partial)$. For example, an analogue of KP theory with matrix coefficients is very difficult. But if we start with (1.19) instead of (1.14), then we can solve it always for an arbitrary initial value of $S$.

The technique we are going to use is a generalization of the Birkhoff decomposition which will be proved in Sect. 3. It is a factorization theorem of an infinite dimensional group. To define the groups, we need micro-differential operators of infinite order. So we define

$$
\begin{aligned}
\widehat{\mathscr{E}}= & \left\{P=\sum_{v \in \mathbf{Z}} a_{v} \partial^{v} \mid a_{v} \in \mathscr{R} \text { and there is a positive real number } C_{P}\right. \text { and positive } \\
& \text { integers } \left.M_{P} \text { and } N_{P} \text { such that } \operatorname{val}_{t} a_{v}>C_{P} v-N_{P} \text { for all } v>M_{P}\right\}
\end{aligned}
$$

and

$$
\widehat{\mathscr{D}}=\left\{P=\sum_{v \in \mathbf{Z}} a_{v} \partial^{v} \mid P \in \widehat{\mathscr{E}} \text { and } a_{v}=0 \text { for } v<0\right\}
$$

Note that $\widehat{\mathscr{E}}$ has a natural associative algebra structure. Let $\mathscr{J}$ be the ideal of $\mathscr{R}$ generated by $\left\{t_{1}, t_{2}, t_{3}, \ldots\right\}$. We denote $\left.P\right|_{t=0}=P \bmod \mathscr{J} \in E$ for every $P \in \hat{\mathscr{E}}$. Lemma 3.1 of Sect. 3 says that if $\left.P\right|_{t=0} \in G$, then $P$ is invertible in $\hat{E}$. Therefore

$$
\widehat{\mathscr{E}}^{\times}=\left\{P \in \widehat{\mathscr{E}}|P|_{t=0} \in G\right\}
$$


forms a group. It is a connected component of the identity operator in the group of all invertible elements of $\hat{E}$. Similarly,

$$
\hat{\mathscr{D}}^{\times}=\left\{P \in \hat{\mathscr{D}}|P|_{t=0}=1\right\}
$$

is a subgroup of $\hat{\mathscr{E}}^{\times}$. The generalized Birkhoff decomposition (Theorem 3.2) says that there is a unique factorization

$$
\begin{aligned}
\widehat{\mathscr{E}}^{\times} & =\mathscr{G} \cdot \hat{\mathscr{D}}^{\times} \\
\omega & \omega \\
U & =S^{-1} \cdot{ }^{\omega} .
\end{aligned}
$$

Let us illustrate how we use this factorization to solve the generalized KP system (1.19). Suppose we have a solution $S \in \mathscr{G}$. Then we also have $Z_{ \pm}=$ $\pm \sum_{n=1}^{\infty} d t_{n}\left(S \partial^{n} S^{-1}\right)_{ \pm}$satisfying $d Z_{ \pm}=Z_{ \pm} \wedge Z_{ \pm}$. Now by Theorem 4.1 of Sect. 4, we have a unique element $Y \in \hat{\mathscr{D}}^{x}$ such that

$$
d Y=Z_{+} Y \text {. }
$$

So define $U=S^{-1} \cdot Y \in \widehat{\mathscr{E}}^{\times}$and let

$$
\Omega=\sum_{n=1}^{\infty} d t_{n} \partial^{n}
$$

Since $d \Omega=0$ and $\Omega \wedge \Omega=0, \Omega$ satisfies the integrability condition

$$
d \Omega=\Omega \wedge \Omega
$$

trivially. Moreover, we have

$$
\begin{aligned}
d U & =-S^{-1} d S \cdot S^{-1} Y+S^{-1} d Y \\
& =S^{-1}\left(d Y \cdot Y^{-1}-d S \cdot S^{-1}\right) S S^{-1} Y \\
& =S^{-1}\left(Z_{+}-Z_{-}\right) S U \\
& =S^{-1} \sum_{n=1}^{\infty} d t_{n} S \partial^{n} S^{-1} S U \\
& =\Omega U,
\end{aligned}
$$

namely, $U=S^{-1} Y$ satisfies the system of linear partial differential equations of constant coefficients;

$$
d U=\Omega U .
$$

Thus a solution $S$ of (1.19) gives rise to a solution $U$ of the universal equation (1.29). Now the factorization theorem (Theorem 3.2) gives a way to go back. So let us start with a solution $U \in \widehat{\mathscr{E}}^{\times}$of the universal equation (1.29). By Theorem 3.2, we have a unique $S \in \mathscr{G}$ and a unique $Y \in \widehat{\mathscr{D}}^{\times}$such that $U=S^{-1} Y$. Let

$$
Z_{ \pm}= \pm \sum_{n=1}^{\infty} d t_{n}\left(S \partial^{n} S^{-1}\right)_{ \pm}
$$


Then

$$
S \Omega S^{-1}=\sum_{n=1}^{\infty} d t_{n}\left(S \partial^{n} S^{-1}\right)=Z_{+}-Z_{-} .
$$

On the other hand, since $d U=\Omega U$, we have

$$
S \Omega S^{-1}=S d U \cdot U^{-1} S^{-1}=d Y \cdot Y^{-1}-d S \cdot S^{-1} .
$$

Comparing $\hat{\mathscr{D}}$ part and $\mathscr{E}^{(-1)}$ part of the above two expressions of $S \Omega S^{-1}$, we obtain $Z_{+}=d Y \cdot Y^{-1}$ and $Z_{-}=d S \cdot S^{-1}$. Namely we have a solution $S$ of Eq. (1.19). If we define $L=S \partial S^{-1}$, then it is a solution of the Lax equation (1.14).

Here comes a natural question. How can we solve the universal equation (1.29)? It is easy. We can solve it for any initial value $\left.U\right|_{t=0}=U(0)$ by simply letting

$$
U(t)=\exp \left(\sum_{n=1}^{\infty} t_{n} \partial^{n}\right) \cdot U(0)
$$

We have chosen our definition (1.21) of $\widehat{\mathscr{E}}$ so that (1.30) gives a well-defined element of $\hat{\mathscr{E}}$ for every $U(0) \in E$. Therefore the operator $\exp \left(\sum_{n=1}^{\infty} t_{n} \partial^{n}\right)$ is the heart of the KP theory. In the next section we generalize it to the case of super derivation.

Now we can show the main theorem of Sect. 1.

Theorem 1.4 (Unique solvability of the generalized KP system). For every initial value $S(0) \in G$, there is a unique solution $S=S(t) \in \mathscr{G}$ of the generalized KP system

$$
d S=-\sum_{n=1}^{\infty} d t_{n}\left(S \partial^{n} S^{-1}\right)_{-} \cdot S
$$

such that $\left.S\right|_{t=0}=S(0)$.

Proof. To establish the existence, we simply define

$$
U=\exp \left(\sum_{n=1}^{\infty} t_{n} \partial^{n}\right) \cdot S(0)^{-1} \in \hat{\mathscr{E}}^{\times} .
$$

Let $U=S^{-1} \cdot Y$ be its unique factorization of (1.25). Then, as we have seen, $S$ gives a solution of (1.19). Since $\left.Y\right|_{t=0}=1$,

$$
S(0)^{-1}=\left.U\right|_{t=0}=\left(\left.S^{-1}\right|_{t=0}\right) \cdot\left(\left.Y\right|_{t=0}\right)=\left(\left.S\right|_{t=0}\right)^{-1},
$$

namely, $\left.S\right|_{t=0}=S(0)$.

To see the uniqueness, suppose we have another solution $\bar{S}$ of (1.19) with the same initial value $\left.\bar{S}\right|_{t=0}=S(0)$. This solution also gives rise to a solution $\vec{U}=\bar{S}^{-1} \cdot \bar{Y}$ of $(1.29)$ with the same initial value $\left.\bar{U}\right|_{t=0}=S(0)^{-1}$. By Lemma 4.3 of Sect. 4, we know the uniqueness for (1.29). Therefore $U=\bar{U}$. But since the decomposition (1.25) is also unique, we conclude $S=\bar{S}$. This completes the proof. 
Remark 1.5. Assume that $R$ is a normed algebra such as a ring of convergent power series in $x$. Then we can establish convergence of the above solution for small $t$. Actually, our construction of the solution $S$ from $S(0)$ and the group factorization (1.25) is explicit (see (3.9)), it is not hard to show the following. Let $S(0)=\sum_{n=0}^{\infty} s_{n} \partial^{-n}$ and the $i$-th derivative of $s_{n}$ satisfy

$$
\left\|s_{n}^{(i)}\right\|<\frac{C^{i} i ! \delta^{n}}{n !}
$$

for a positive constant $C$ and a small $\delta>0$ for all $n$ and $i$. Then the above solution $S \in \mathscr{G}$ converges absolutely for all $t=\left(t_{1}, t_{2}, t_{3}, \ldots\right)$ satisfying

$$
\left|t_{n}\right|<A \cdot \frac{\varepsilon^{n}}{n !}
$$

for an arbitrary positive number $A$ and a sufficiently small $\varepsilon>0$. (For more detail, please see my forthcoming book on KP equations.)

Remark 1.6. Let us consider the case of $R=\mathbb{C}((x))[\log x]$. Since it satisfies the exactness of (1.20), the Lax equation (1.14) or (1.9) is uniquely solvable for any initial value

$$
L(0)=\partial+u_{2} \partial^{-1}+u_{3} \partial^{-2}+\ldots \in E
$$

with coefficients in $\mathbb{C}((x))[\log x]$. Namely, every ordinary differential operator with arbitrary poles and logarithmic singularities at the origin $x=0$ has also a unique universal family of isospectral deformations. In the case of the $\mathrm{KdV}$ equation, it means that there exists a unique solution $u(x, t) \in(\mathbb{C}((x))[\log x]) \llbracket t \rrbracket$ of the $\mathrm{KdV}$ equation

$$
u_{t}=\frac{1}{4} u_{x x x}+3 u u_{x}
$$

with an initial condition $u(x, 0)=f(x)$ for every given function $f(x) \in \mathbb{C}((x))[\log x]$. Our expression for $u(x, t)$ obtained by the above theorem gives an asymptotic expansion of the solution around the singularity at $x=0$.

Now an interesting thing happens. The space of all solutions of (1.19) for $R=\mathbb{C}((x))[\log x]$ actually contains Sato's universal Grassmann manifold (UGM) [11] and Segal-Wilson's Grassmannian of the loop space [12] as proper subspaces. Let $G_{R}$ denote the group of (1.16) defined over an algebra $R$. By the unique solvability of the Cauchy problem, $G_{R}$ is the solution space for the generalized KP system (1.9). It is easy to see that $G_{C[x \rrbracket]}$ is an affine open subset (actually the "big-cell") of Sato's UGM. On the other hand, UGM is a subset of an affine space $G_{\mathbf{C}((x))[\log x]}$;

$$
G_{\mathbf{C} \llbracket x \rrbracket} \subset U G M \subset G_{\mathbf{C}((x))[\log x]} .
$$

Since all the compact complex Grassmann varieties are embedded in UGM, they are also embedded in our affine space!

Sato's UGM can be regarded as a subspace of the solution space $G_{\mathbf{C}((x))[\log x]}$ consisting of operators $S$ such that the corresponding $L=S \partial S^{-1}$ has a regular singularity at $x=0$. 
Remark 1.7. Since the basic differential algebra $(R, \partial)$ is arbitrary in Theorem 1.4, we can take

$$
R=\left\{\sum_{v \in \mathbf{Z}} a_{v}(x, y)\left(\frac{d}{d y}\right)^{v} \mid a_{v}=0 \text { for } v \gg 0\right\}
$$

and $\partial=d / d x$. In other words, we can consider something line a $\mathrm{KP} \otimes \mathrm{KP}$ system. This example gives us a way to construct a higher dimensional analogue of the KP theory.

\section{The super KP system}

We introduce the super KP system in a rather different manner from Manin and Radul [6]. We will follow the argument of Sect. 1 in the reverse direction. So we start with the universal equation of the form $d U=\Omega U$ and then define the super KP system by using a group decomposition. After this, we will also give the Lax formalism for the super KP system.

To make our presentation precise, let us introduce algebras and groups of operators we need. Let $(R, \theta)$ be a super-differential algebra defined over a field $k$ of characteristic zero. Namely, $R=R_{0} \oplus R_{1}$ is a $Z_{2}$-graded associative $k$-algebra with unity (called a superalgebra) and $\theta: R \rightarrow R$ is a $k$-linear map satisfying the super Leibnitz rule

$$
\theta(a b)=\theta(a) \cdot b+(-1)^{\tilde{a}} a \theta(b),
$$

where $b \in R$ and $a$ is a homogeneous element of $R$ with $\mathbf{Z}_{2}$-degree $\tilde{a} \in \mathbf{Z}_{2}$. Such a map $\theta$ is called an odd super-derivation. We also define $\tilde{n}=1 / 2\left(1-(-1)^{n}\right) \in \mathbf{Z}_{2}$ for $n \in \mathbf{Z}$. The super algebra $R$ is said to be super-commuting if

$$
a b=(-1)^{a \tilde{b}} b a
$$

for all homogeneous elements $a$ and $b$ of $R$. We do not assume super-commutativity of $R$.

We define the set of all super micro-differential operators

$$
E=\left\{P=\sum_{v \in \mathbf{Z}} a_{v} \theta^{v} \mid a_{v} \in R \text { and } a_{v}=0 \text { for } v \gg 0\right\},
$$

and the set of super differential operators

$$
D=\left\{P=\sum_{\nu \in \mathbf{Z}} a_{\nu} \theta^{\nu} \mid P \in E \text { and } a_{v}=0 \text { for } v<0\right\} .
$$

To introduce associative algebra structures in $E$ and $D$, we need the super binomial coefficients $\left[\begin{array}{l}v \\ i\end{array}\right]$. If $v$ and $i$ are both in $\mathbf{N}=\{0,1,2, \ldots\}$, then we define $\left[\begin{array}{l}v \\ i\end{array}\right]$ by

$$
(a+b)^{v}=\sum_{i=0}^{\infty}\left[\begin{array}{l}
v \\
i
\end{array}\right] a^{i} b^{v-i}
$$


where $a$ and $b$ are super-commuting homogeneous elements of $\mathbf{Z}_{2}$-degree $1 \in \mathbf{Z}_{2}$. For $v \in \mathbf{Z}$ and $i \in \mathbf{N}$, we define

$$
\left\{\begin{array}{l}
{\left[\begin{array}{l}
v \\
i
\end{array}\right]=0 \quad \text { if } 0 \leqq v<i \quad \text { or } \quad(v, i) \equiv(0,1) \bmod 2,} \\
{\left[\begin{array}{l}
v \\
i
\end{array}\right]=\left(\begin{array}{c}
{[v / 2]} \\
{[i / 2]}
\end{array}\right) \quad \text { if } v<0 \text { and }(v, i) \neq(0,1) \bmod 2}
\end{array}\right.
$$
where $[v]$ denotes the largest integer not greater than $v$. We can extend $\left[\begin{array}{l}v \\ i\end{array}\right]$
for negative $i$ by using

$$
\left[\begin{array}{c}
v \\
v-i
\end{array}\right]=\left[\begin{array}{l}
v \\
i
\end{array}\right] \text { and }\left[\begin{array}{l}
v \\
i
\end{array}\right]+(-1)^{i+1}\left[\begin{array}{c}
v \\
i+1
\end{array}\right]=\left[\begin{array}{c}
v+1 \\
i+1
\end{array}\right]
$$
however, we need only $\left[\begin{array}{l}v \\ i\end{array}\right]$ with $v \in \mathbf{Z}$ and $i \in \mathbf{N}$. Let us give actual values for
small $i$ :

$$
\begin{aligned}
& {\left[\begin{array}{l}
v \\
0
\end{array}\right]=1} \\
& {\left[\begin{array}{l}
v \\
1
\end{array}\right]=\tilde{v}=\frac{1}{2}\left(1-(-1)^{v}\right)} \\
& {\left[\begin{array}{l}
v \\
2
\end{array}\right]=\left[\frac{v}{2}\right]} \\
& {\left[\begin{array}{l}
v \\
3
\end{array}\right]= \begin{cases}0 & \text { if } v \text { is even } \\
{\left[\frac{v}{2}\right]=\frac{v-1}{2}} & \text { if } v \text { is odd }\end{cases} } \\
& {\left[\begin{array}{l}
v \\
4
\end{array}\right]=\frac{\left[\frac{v}{2}\right]\left(\left[\frac{v}{2}\right]-1\right)}{2} .}
\end{aligned}
$$

We can introduce an associative algebra structure in $E$ by using the general super Leibnitz rule

$$
\theta^{v} \cdot f=\sum_{i=0}^{\infty}\left[\begin{array}{l}
v \\
i
\end{array}\right](-1)^{\tilde{f}(\nu-i)} f^{[i]} \theta^{v-i}
$$

for $v \in \mathbf{Z}$ and $f \in R$, where $f^{[i]}=\theta^{i}(f)$. We also have an algebra decomposition

$$
E=E^{(-1)} \oplus D
$$

in an obvious way, where $E^{(-1)}=\left\{P=\sum_{v \in Z} a_{v} \theta^{v} \mid a_{v}=0\right.$ for $\left.v>0\right\}$. Every $P \in E$ decomposes into $P=P_{-} \oplus P_{+}$with $P_{-} \in E^{(-1)}$ and $P_{+} \in D$. The algebra $E$ has 
also a super algebra structure defined by

$$
\left\{\begin{aligned}
E & =E_{0} \oplus E_{1}, \\
E_{0} & =\left\{P=\sum_{v \in \mathbf{Z}} a_{v} \theta^{v} \mid P \in E \text { and } \tilde{a}_{v}=\tilde{v} \in \mathbf{Z}_{2}\right\} \\
E_{1} & =\left\{P=\sum_{v \in \mathbf{Z}} a_{v} \theta^{v} \mid P \in E \text { and } \tilde{a}_{v}=(\widetilde{v+1}) \in \mathbf{Z}_{2}\right\}
\end{aligned}\right.
$$

Similarly, we define $D_{0}=D \cap E_{0}$ and $D_{1}=D \cap E_{1}$ to make $D$ a super algebra. The above $E_{0}$ is the set of homogeneous even operators and $E_{1}$ is the set of homogeneous odd operators. The group $G$ of inner automorphisms is defined by

$$
G=\left\{S=\sum_{n=0}^{\infty} s_{n} \theta^{-n} \mid s_{0}=1, s_{n} \in R\right\} .
$$

Its even part is given by

$$
G_{0}=\left\{S=\sum_{n=0}^{\infty} s_{n} \theta^{-n} \mid S \in G \text { and } \tilde{s}_{n}=\tilde{n} \in \mathbf{Z}_{2}\right\}
$$

but the odd part is not a group since $\tilde{s}_{0}=0$ in $G$.

As in Sect. 1 we use infinitely many deformation parameters $t_{1}, t_{2}, t_{3}, t_{4}, \ldots$ We define

$$
\mathscr{R}=R\left[t_{1}, t_{2}, t_{3}, \ldots \rrbracket\right.
$$

and val $_{t}: \mathscr{R} \backslash\{0\} \rightarrow \mathbf{N}$ as before, namely we set $\operatorname{val}_{l}\left(t_{n}\right)=n \in \mathbf{N}$. However, the $\mathbf{Z}_{2}$-degree of $t_{n}$ is defined to be

$$
\tilde{t}_{n}=\tilde{n} \in \mathbf{Z}_{2}
$$

Namely, all even variables $t_{2}, t_{4}, t_{6}, \ldots$ are usual commutative variables, while odd variables $t_{1}, t_{3}, t_{5}, \ldots$ are Grassmann variables satisfying

$$
\left\{\begin{aligned}
t_{2 n-1} t_{2 m-1} & =-t_{2 m-1} t_{2 n-1} \\
\left(t_{2 n-1}\right)^{2} & =0
\end{aligned}\right.
$$

The $t$-depending operators we consider are the elements of

$$
\mathscr{E}=\left\{P=\sum_{v \in \mathbf{Z}} a_{v} \theta^{v} \mid a_{v} \in R \text { and } a_{v}=0 \text { for } v \gg 0\right\}
$$

Similarly, we introduce $\mathscr{D}, \mathscr{E}^{(-1)}, \mathscr{D}_{0}, \mathscr{D}_{1}, \mathscr{E}_{0}, \mathscr{E}_{1}, \mathscr{G}$ and $\mathscr{G}_{0}$ in an obvious way as before. The completion of $\mathscr{E}$ is also defined similarly;

$$
\widehat{E}=\left\{P=\sum_{v \in \mathbf{Z}} a_{v} \theta^{v} \mid a_{v} \in \mathscr{R} \text { and there is a positive real number } C_{P}\right. \text { and positive }
$$

integers $M_{P}$ and $N_{P}$ such that val $a_{v}>C_{P} v-N_{P}$ for all $\left.v>M_{P}\right\}$. 
The completion $\hat{\mathscr{D}}$ of $\mathscr{D}$ is also given by the same way. Note that $\hat{\mathscr{E}}$ has a natural associative algebra structure as well as a super algebra structure $\hat{\mathscr{E}}=\widehat{\mathscr{E}}_{0} \oplus \hat{\mathscr{E}}_{1}$.

As in Sect. 1, let $\mathscr{J}$ be the ideal of $\mathscr{R}$ generated by $\left\{t_{1}, t_{2}, t_{3}, t_{4}, \ldots\right\}$ and we write

$$
\left.P\right|_{t=0}=P \bmod \mathscr{J} \in E \quad \text { for all } P \in \widehat{\mathscr{E}} \text {. }
$$

Lemma 3.3 of Sect. 3 says that $P \in \hat{\mathscr{E}}$ is invertible if $\left.P\right|_{t=0} \in G$. So we set

$$
\begin{aligned}
& \mid \widehat{\mathscr{E}} \times=\left\{P \in \widehat{\mathscr{E}}|P|_{t=0} \in G\right\} \\
& \mid \hat{\mathscr{D}}^{\times}=\left\{P \in \mathscr{\mathscr { D }}|P|_{t=0}=1\right\} .
\end{aligned}
$$

These are infinite dimensional groups. We also introduce

$$
\widehat{\mathscr{E}}_{0}^{\times}=\widehat{\mathscr{E}}_{0} \cap \hat{\mathscr{E}}^{\times} \text {and } \hat{\mathscr{D}}^{\times}=\hat{\mathscr{D}}_{0} \cap \hat{\mathscr{D}}^{\times} \text {. }
$$

The generalized super Birkhoff decomposition (Theorem 3.4) gives the unique factorizations

and

$$
\begin{gathered}
\hat{\mathscr{E}}^{\times}=\mathscr{G} \cdot \hat{\mathscr{D}}^{\times} \\
\stackrel{\omega}{U}=\stackrel{\omega}{S^{-1} \cdot \stackrel{\omega}{Y}^{\prime}}
\end{gathered}
$$

$$
\begin{aligned}
\hat{\mathscr{E}}_{0}^{\times} & =\mathscr{G}_{0} \cdot \hat{\mathscr{D}}_{0}^{\times} \\
\stackrel{\omega}{\omega} & \stackrel{\omega}{S_{0}^{-1}} \cdot \stackrel{\omega}{Y_{0} .} .
\end{aligned}
$$

With these preparations, we can now introduce the universal equation for the super KP system. Consider the following element of $\hat{\mathscr{E}}_{0} \times$;

$$
H=\exp \left(\sum_{n=1}^{\infty} t_{n} \theta^{n}\right)
$$

This is the super extension of the important operator $\exp \left(\sum_{n=1}^{\infty} t_{n} \partial^{n}\right)$ of (1.30) and gives us the basic motivation how to generalize the KP system into the super category. If we set

$$
A=\sum_{n=1}^{\infty} t_{2 n} \theta^{2 n} \text { and } B=\sum_{n=1}^{\infty} t_{2 n-1} \theta^{2 n-1},
$$

then $B^{2}=0$ and

$$
H=e^{A+B}=e^{A} e^{B}=e^{B} e^{A}=(1+B) e^{A} .
$$

From this we can see that $H$ is a well-defined operator in $\widehat{\mathscr{E}}_{0}^{\times}$. Now define

$$
\Omega=d H \cdot H^{-1},
$$


where $d=\sum_{n=1}^{\infty} d t_{n} \partial / \partial t_{n}$ is the super $t$-exterior differentiation. (Therefore $d t_{2 n}$ and $d t_{2 m}$ anti-commute while $d t_{2 n-1}$ and $d t_{2 m-1}$ commute.) Using (2.17) we get

$$
\begin{aligned}
\Omega & =d\left((1+B) e^{A}\right) e^{-A}(1-B)=(1+B) d A(1-B)+d B(1-B) \\
& =d A+d B(1-B) .
\end{aligned}
$$

Therefore

$$
d \Omega=d B \wedge d B
$$

and

$$
\begin{aligned}
\Omega \wedge \Omega= & d A \wedge d A+d A \wedge d B(1-B)+d B(1-B) \wedge d A+d B(1-B) \\
& \wedge d B(1-B)=d B \wedge d B .
\end{aligned}
$$

Namely, $\Omega$ satisfies the integrability condition

$$
d \Omega=\Omega \wedge \Omega .
$$

Note that here is an important difference between the super KP system and the usual KP system. In the latter, remember that we have $\Omega$ which satisfies (1.28) trivially. But in the super case, (2.18) does not satisfy $d \Omega=0$ nor $\Omega \wedge \Omega=0$.

The explicit form of $\Omega$ is given by

$$
\Omega=\sum_{n=1}^{\infty} d t_{2 n} \theta^{2 n}+\sum_{n=1}^{\infty} d t_{2 n-1} \theta^{2 n-1}\left(1-\sum_{k=1}^{\infty} t_{2 k-1} \theta^{2 k-1}\right) .
$$

Consider the universal equation

$$
d U=\Omega U
$$

for $U \in \widehat{\mathscr{E}}_{0}$. It is obvious from the definition of $\Omega$ that $U=H \cdot U(0)$ is a solution for every $U(0)=\left.U\right|_{t=0} \in E_{0}$. The uniqueness of the Cauchy problem for (2.21) is proved in Sect. 4 (see Lemma 4.4).

Take an arbitrary element $S(0) \in G_{0}$. Then $U=H \cdot S(0)^{-1}$ is a well-defined operator in $\hat{\mathscr{E}}_{0}^{\times}$and solves (2.21). Let $U=S^{-1} \cdot Y$ be the decomposition of $U$ according to (2.15). Then

$$
S \Omega S^{-1}=S d U U^{-1} S^{-1}=-d S \cdot S^{-1}+d Y \cdot Y^{-1} .
$$

Comparing $\mathscr{E}_{0}^{(-1)}$ part and $\hat{\mathscr{D}}_{0}$ part of the above, we obtain

where we define

$$
\begin{gathered}
d S \cdot S^{-1}=Z_{-} \\
d Y \cdot Y^{-1}=Z_{+},
\end{gathered}
$$

$$
\begin{aligned}
Z_{ \pm}= \pm & \left(\sum_{n=1}^{\infty} d t_{2 n}\left(S \theta^{2 n} S^{-1}\right)_{ \pm}+\sum_{n=1}^{\infty} d t_{2 n-1}\left(S \theta^{2 n-1} S^{-1}\right)_{ \pm}\right. \\
& \left.+\sum_{n=1}^{\infty} \sum_{k=1}^{\infty} d t_{2 n-1} \cdot t_{2 k-1}\left(S \theta^{2 n-1+2 k-1} S^{-1}\right)_{ \pm}\right)
\end{aligned}
$$


Motivated by the above expressions, we call the following equation the super KP system;

$$
\begin{aligned}
d S=- & \left(\sum_{n=1}^{\infty} d t_{n}\left(S \theta^{n} S^{-1}\right)_{-}\right. \\
& \left.+\sum_{n=1}^{\infty} \sum_{k=1}^{\infty} d t_{2 n-1} \cdot t_{2 k-1}\left(S \theta^{2 n+2 k-2} S^{-1}\right)_{-}\right) \cdot S
\end{aligned}
$$

To obtain the Lax equation, we define

$$
L=S \theta S^{-1}
$$

which is no longer a normalized operator. It is a monic operator of the form

$$
L=\theta+u_{1}+u_{2} \theta^{-1}+u_{3} \theta^{-2}+\ldots
$$

with $\tilde{u}_{n}=\tilde{n} \in \mathbf{Z}_{2}$. The first two coefficients satisfy $u_{1}^{[1]}+2 u_{2}=-u_{1}^{2}$.

Then Eq. (2.25) gives the Lax equation in terms of $L$;

$$
d L=\left[Z_{-}, L\right]
$$

where $[$,$] denotes the super Lie bracket defined by [a, b]=a b-(-1)^{a \tilde{b}} b a$ for homogeneous elements. Since $S \Omega S^{-1}=Z_{+}-Z_{-}$, we have

Therefore

$$
d L=\left[Z_{+}-S \Omega S^{-1}, L\right] \text {. }
$$

$$
\left\{\begin{aligned}
\frac{\partial L}{\partial t_{2 n}} & =\left[\left(L^{2 n}\right)_{+}, L\right] \\
\frac{\partial L}{\partial t_{2 n-1}} & =\left[\left(L^{2 n-1}\right)_{+}, L\right]-\left[L^{2 n-1}, L\right]+\left[\sum_{k=1}^{\infty} t_{2 k-1}\left(L^{2 n+2 k-2}\right)_{+}, L\right] \\
& =\left[\left(L^{2 n-1}\right)_{+}, L\right]-2 L^{2 n}+\sum_{k=1}^{\infty} t_{2 k-1}\left[\left(L^{2 n+2 k-2}\right)_{+}, L\right]
\end{aligned}\right.
$$

This system of equations is called the Lax equation for the super KP system. Actually it is equivalent to the system of Manin and Radul [6]. To obtain their equations, simply change $t_{n}$ to $-t_{n}$. Remember that our motivation of introducing the super KP system was to replace $\exp \left(\sum_{n=1}^{\infty} t_{n} \partial^{n}\right)$ of $(1.30)$ by $\exp \left(\sum_{n=1}^{\infty} t_{n} \theta^{n}\right)$ of (2.16). If we define $\theta_{2 n}=\partial / \partial t_{2 n}$ and $\theta_{2 n-1}=\partial / \partial t_{2 n-1}$ $-\sum_{k=1}^{\infty} t_{2 k-1} \partial / \partial t_{2 k+2 n-2}$, then they satisfy $\left[\theta_{2 n}, \theta_{2 m}\right]=0$ and $\left[\theta_{2 n-1}, \theta_{2 m-1}\right]=$ $-2 \theta_{2 n+2 m-2}$. On the other hand, Manin and Radul want to have $\left[\theta_{2 n-1}, \theta_{2 m-1}\right]=2 \theta_{2 n+2 m-2}$ and use this as their motivation. 
Our main theorem of this section is the following;

Theorem 2.1 (unique solvability of the super KP system). For every given element $S(0) \in G_{0}$, there exists a solution $S \in \mathscr{G}_{0}$ of the super $K P$ system

$$
\begin{aligned}
d S=- & \left(\sum_{n=1}^{\infty} d t_{n}\left(S \theta^{n} S^{-1}\right)_{-}\right. \\
& \left.+\sum_{n=1}^{\infty} \sum_{k=1}^{\infty} d t_{2 n-1} \cdot t_{2 k-1}\left(S \theta^{2 n+2 k-2} S^{-1}\right)_{-}\right) \cdot S
\end{aligned}
$$

with the initial value $\left.S\right|_{t=0}=S(0)$. Moreover, if $(R, \theta)$ satisfies the Condition 4.5 of Sect. 4 , then the above solution is unique.

Proof. We start with $U=H \cdot S(0)^{-1} \in \hat{\mathscr{E}}_{0}^{X}$ and decompose it as $U=S^{-1} \cdot Y$ according to (2.15). Then $S$ solves (2.25) and satisfies $\left.S\right|_{t=0}=S(0)$. The uniqueness follows from the facts that every solution of (2.25) gives rise to a solution of the universal equation (2.21) (see Theorem 4.6), its uniqueness (Lemma 4.4) and the uniqueness of the factorization (Theorem 3.4). This completes the proof.

For a general super differential algebra $(R, \theta)$, we cannot say much about solvability of the Lax equation (2.27). However, if we assume an appropriate condition on $(R, \theta)$ and restrict a class of $L$, then we can also establish the unique solvability for (2.27).

Since (2.25) is solvable, it is natural to try to find an $S \in G_{0}$ for a given $L$. So let us characterize a first order monic operator $L \in E_{1}$ such that $L=S \theta S^{-1}$ for some $S \in G_{0}$. Let

$$
\begin{aligned}
S & =1+s_{1} \theta^{-1}+s_{2} \theta^{-2}+\ldots, \\
S^{-1} & =1+w_{1} \theta^{-1}+w_{2} \theta^{-2}+\ldots, \quad \text { and } \\
L & =\theta+u_{1}+u_{2} \theta^{-1}+\ldots .
\end{aligned}
$$

From $S \cdot S^{-1}=1$ we obtain $s_{1}+w_{1}=0$ and $s_{2}-s_{1} w_{1}+w_{2}=0$. The equation $L$ $=S \theta S^{-1}$ implies that $u_{1}=2 s_{1}$ and $u_{2}=-1 / 2\left(u_{1}^{2}+u_{1}^{[1]}\right)$. Therefore $u_{1}$ and $u_{2}$ satisfy

$$
\frac{1}{2} u_{1}^{2}+\frac{1}{2} u_{1}^{[1]}+u_{2}=0 .
$$

What is remarkable here is that the converse is essentially true.

Proposition 2.2. Let $(R, \theta)$ be a super differential algebra such that

$$
\theta^{2}: R \rightarrow R \quad \text { is surjective. }
$$

Then for a monic first order odd operator

$$
L=\theta+u_{1}+u_{2} \theta^{-1}+u_{3} \theta^{-2}+\ldots \in E_{1},
$$


there exists an $S \in G_{0}$ such that

$$
L=S \theta S^{-1}
$$

if and only if $u_{1}$ and $u_{2}$ satisfy

$$
\frac{1}{2} u_{1}^{2}+\frac{1}{2} u_{1}^{[1]}+u_{2}=0 .
$$

Proof. Our idea is to solve the equation $L S-S \theta=0$ for $S$. Let $L=\sum_{n=0}^{\infty} u_{n} \theta^{1-n}$, $\tilde{u}_{n}=\tilde{n} \in \mathbf{Z}_{2}$ and $u_{0}=1$, and $S=\sum_{m=0}^{\infty} s_{m} \theta^{-m}, \tilde{s}_{m}=\tilde{m} \in \mathbf{Z}_{2}$ and $s_{0}=1$. Then

$$
\begin{aligned}
0 & =L S-S \theta=\left(\sum_{n=0}^{\infty} u_{n} \theta^{1-n}\right)\left(\sum_{m=0}^{\infty} s_{m} \theta^{-m}\right)-\sum_{m=0}^{\infty} s_{m} \theta^{1-m} \\
& =\sum_{n=0}^{\infty} \sum_{m=0}^{\infty} \sum_{i=0}^{\infty}\left[\begin{array}{c}
1-n \\
i
\end{array}\right](-1)^{m(1-n-i)} u_{n} s_{m}^{[i]} \theta^{1-n-m-i}-\sum_{\alpha=0}^{\infty} s_{\alpha} \theta^{1-\alpha} \\
& =\sum_{\alpha=0}^{\infty}\left(\sum_{m=0}^{\alpha} \sum_{i=0}^{\alpha-m}\left[\begin{array}{c}
1-\alpha+m+i \\
i
\end{array}\right](-1)^{m \alpha} u_{\alpha-m-i} s_{m}^{[i]}-s_{\alpha}\right) \theta^{1-\alpha}
\end{aligned}
$$

Let $\alpha=2 n$. Then

$$
\begin{aligned}
0 & =\sum_{m=0}^{2 n} \sum_{i=0}^{2 n-m}\left[\begin{array}{c}
1-2 n+m+i \\
i
\end{array}\right] u_{2 n-m-i} s_{m}^{[i]}-s_{2 n} \\
& =\sum_{m=0}^{2 n-1} \sum_{i=0}^{2 n-m}\left[\begin{array}{c}
1-2 n+m+i \\
i
\end{array}\right] u_{2 n-m-i} s_{m}^{[i]} \\
& =s_{2 n-1}^{[1]}+u_{1} s_{2 n-1}+\sum_{m=0}^{2 n-2} \sum_{i=0}^{2 n-m-2}\left[\begin{array}{c}
1-2 n+m+i \\
i
\end{array}\right] u_{2 n-m-i} s_{m}^{[i]} .
\end{aligned}
$$

If $\alpha=2 n+1$, then

$$
\begin{aligned}
0= & \sum_{m=0}^{2 n+1} \sum_{i=0}^{2 n+1-m}\left[\begin{array}{c}
-2 n+m+i \\
i
\end{array}\right](-1)^{m} u_{2 n+1-m-i} s_{m}^{[i]}-s_{2 n+1} \\
& =-2 s_{2 n+1}+s_{2 n}^{[1]}+u_{1} s_{2 n}+\sum_{m=0}^{2 n-1} \sum_{i=0}^{2 n-m-1}\left[\begin{array}{c}
-2 n+m+i \\
i
\end{array}\right](-1)^{m} u_{2 n+1-m-i} s_{m}^{[i]} .
\end{aligned}
$$

Thus we have

$$
\begin{aligned}
s_{2 n+1}= & \frac{1}{2} s_{2 n}^{[1]}+\frac{1}{2} u_{1} s_{2 n} \\
& +\frac{1}{2} \sum_{m=0}^{2 n-1} \sum_{i=0}^{2 n-m-1}\left[\begin{array}{c}
-2 n+m+i \\
i
\end{array}\right](-1)^{m} u_{2 n+1-m-i} s_{m}^{[i]}
\end{aligned}
$$


Using this expression with the even case above, we get

$$
\begin{aligned}
& \frac{1}{2} s_{2 n}^{[2]}+\frac{1}{2} u_{1}^{2} s_{2 n}+\frac{1}{2} u_{1}^{[1]} s_{2 n}+u_{2} s_{2 n} \\
& \quad+\sum_{m=0}^{2 n-i} \sum_{i=0}^{2 n-m-i}\left\{\left[\begin{array}{c}
m-1-2 n+i \\
i
\end{array}\right] u_{2 n+2-m-i} s_{m}^{[i]}\right. \\
& \left.\quad+\frac{1}{2}\left[\begin{array}{c}
m-2 n+i \\
i
\end{array}\right](-1)^{m}\left(\left(u_{2 n+1-m-i} s_{m}^{[i]}\right)^{[1]}+u_{1} u_{2 n+1-m-i} s_{m}^{[i]}\right)\right\}=0 .
\end{aligned}
$$

Because of the assumption (2.28), we have an recursion formula

$$
\begin{aligned}
s_{2 n}^{[2]}= & -\sum_{m=0}^{2 n-1} \sum_{i=0}^{2 n-m-1}\left\{2\left[\begin{array}{c}
m-1-2 n+i \\
i
\end{array}\right] u_{2 n+2-m-i} s_{m}^{[i]}\right. \\
& \left.+\left[\begin{array}{c}
m-2 n+i \\
i
\end{array}\right](-1)^{m}\left(\left(u_{2 n+1-m-i} s_{m}^{[i]}\right)^{[1]}+u_{1} u_{2 n+1-m-i} s_{m}^{[i]}\right)\right\} .
\end{aligned}
$$

Since $\theta^{2}: R \rightarrow R$ is surjective, (2.30) and (2.31) determine all $s_{n}$ 's up to constants of integration. Therefore $S \in G_{0}$ exists.

Theorem 2.3. Let $(R, \theta)$ be a super-commutative super-differential algebra satisfying Condition 4.5 and that $\theta^{2}: R \rightarrow R$ is surjective. Then for every monic first order odd operator $L(0) \in E_{1}$ with the condition (2.28), there exists a unique solution $L \in \mathscr{E}_{1}$ of the Lax equation (2.27) such that $\left.L\right|_{t=0}=L(0)$.

Proof. The existence is obvious from Theorem 2.1 and Proposition 2.2. To establish uniqueness, we want to show that every solution $L$ of (2.27) starting with $\left.L\right|_{t=0}=L(0)$ satisfies the condition (2.28) for all $t$. Assume that it is acrually true. Let $S(0) \in G_{0}$ be such that $L(0)=S(0) \theta S(0)^{-1}$ and $S \in \mathscr{G}_{0}$ the unique solution of (2.25) starting at $S(0)$. Then $L=S \theta S^{-1}$ is a solution of (2.27) with initial value $\left.L\right|_{t=0}=L(0)$. Now suppose that $L \in \mathscr{E}_{1}$ is another solution of (2.27) with the same initial value $\left.\bar{L}\right|_{t=0}=L(0)$. Since we are assuming that $\bar{L}$ satisfies (2.28), there exists an operator $\bar{S} \in \mathscr{G}_{0}$ such that $\bar{L}=\bar{S} \theta \bar{S}^{-1}$. Unfortunately, such an $\bar{S}$ is not unique. However, if we impose $\bar{S}$ to be a solution of $(2.25)$, then it is uniquely determined by its initial value $\bar{S}(0) \in G_{0}$. Note that

$$
L(0)=S(0) \theta S(0)^{-1}=\bar{S}(0) \theta \bar{S}(0)^{-1} .
$$

Therefore $C=\bar{S}(0)^{-1} S(0) \in G_{0}$ satisfies $C \theta=\theta C$. Let $\bar{U}=H \cdot \bar{S}(0)^{-1}$ and $U$ $=H \cdot S(0)^{-1}$. Then

$$
\begin{aligned}
U & =H \cdot S(0)^{-1}=H \cdot(\bar{S}(0) C)^{-1}=H C^{-1} \bar{S}(0)^{-1} \\
& =(1+B) e^{A} C^{-1} \bar{S}(0)^{-1}=C^{-1} H \bar{S}(0)^{-1}=C^{-1} \bar{U} .
\end{aligned}
$$

If $U=S^{-1} \cdot Y$ and $\bar{U}=\bar{S}^{-1} \cdot \bar{Y}$ be the decomposition, then $C^{-1} \bar{S}^{-1}=S^{-1}$ because of the uniqueness. Therefore $S=\bar{S} C$. But then we have

$$
L=S \theta S^{-1}=\bar{S} C \theta(\bar{S} C)^{-1}=\bar{S} C \theta C^{-1} \bar{S}^{-1}=\bar{S} \theta \bar{S}^{-1}=\bar{L} .
$$

This establishes the uniqueness. 
The only thing we have to show is that (2.27) implies (2.28) if its initial value satisfies (2.28). Let $r_{n+1}=\operatorname{Res}\left(L^{n}\right)=$ the coefficient of $\theta^{-1}$ in the expansion of $L^{n}$. Note that $\tilde{r}_{n}=\tilde{n} \in \mathbf{Z}_{2}$. Then (2.27) implies that

$$
\left\{\begin{array}{cl}
\frac{\partial u_{1}}{\partial t_{2 n}}=-2 r_{2 n+1}, & \frac{\partial u_{2}}{\partial t_{2 n}}=r_{2 n+1}^{[1]}+r_{2 n+1} u_{1}+u_{1} r_{2 n+1}, \\
\frac{\partial u_{1}}{\partial t_{2 n-1}}=-2 r_{2 n}, & \frac{\partial u_{2}}{\partial t_{2 n-1}}=-r_{2 n}^{[1]}+r_{2 n} u_{1}-u_{1} r_{2 n} .
\end{array}\right.
$$

Since we assumed that $R$ is super-commutative, we obtain

$$
\frac{\partial}{\partial t_{n}}\left(u_{1}^{[1]}+2 u_{2}\right)=0
$$

for all $n=1,2,3,4, \ldots$. Note that $u_{1}^{2}=0$ in a super-commutative algebra. Since $\left.\left(u_{1}^{[1]}+2 u_{2}\right)\right|_{t=0}=0$ by assumption, (2.32) implies that $u_{1}^{[1]}+2 u_{2}=0$ as an element of $\mathscr{R}$. This completes the proof of Theorem 2.3.

\section{Generalized Birkhoff Decomposition}

In the previous sections we observed that the groups of infinite order (super) micro-differential operators play a central role in establishing the unique solvability of the initial value problem for the (super) KP systems. In this section we give a proof of the decompositions (1.25), (2.14) and (2.15).

In the first half part of this section, $\hat{\mathscr{E}}^{x}$ denotes the set introduced in (1.23). We start with the following;

Lemma 3.1. Every element $P \in \widehat{\mathscr{E}}^{\times}$is invertible in $\hat{\mathscr{E}}^{\times}$. More precisely, the Neumann series $\sum_{n=0}^{\infty}(1-P)^{n}$ gives an well-defined element in $\hat{\mathscr{E}}^{\times}$, which we define $P^{-1}$.

Proof. Let $P=\sum_{v \in \mathbf{Z}} a_{v} \partial^{v}, a_{v} \in \mathscr{R}$. Since $P \in \widehat{\mathscr{E}}$, its coefficients satisfy a growth order condition

$$
\text { val }_{t} a_{v}>c_{P} v-N_{P} \quad \text { for all } v>M_{P} \text {. }
$$

Let $Q=1-P=\sum_{v \in \mathbf{Z}} b_{v} \partial^{v} \in \widehat{\mathscr{E}}$. Since $\left.Q\right|_{t=0}=1-\left.P\right|_{t=0} \in E^{(-1)}$, val ${ }_{t} b_{v} \geqq 1$ for all $v \geqq 0$.

Of course the coefficients of $Q$ satisfy the same growth order condition

$$
\operatorname{val}_{t} b_{v}>c_{P} v-N_{P} \text { for all } v>M_{P} .
$$

Thus there exists a positive real number $J$ such that

$$
\operatorname{val}_{t} b_{v} \geqq J(v+1) \quad \text { for all } v \geqq-1 .
$$

Actually, $J=c_{P} /\left(1+c_{P} M_{P}+N_{P}\right)$ will do.

Let $Q^{n}=\sum_{v \in \mathbf{Z}} b_{n, v} \partial^{v} \in \hat{\mathscr{E}}$. Then we have;

Claim. $\operatorname{val}_{t} b_{n, v} \geqq J(v+n)$ for all $v \geqq-n$. 
Proof of the Claim. We use an induction on $n$. If $n=1$, then $b_{1, v}=b_{v}$ and (3.2) reduces to (3.1). Now assume that (3.2) is true for some $n$. We want to show $\mathrm{val}_{t} b_{n+1, v} \geqq J(v+n+1)$ for $v \geqq-n-1$. Since

$$
\begin{aligned}
Q^{n+1} & =Q \cdot Q^{n}=\sum_{\alpha \in \mathbf{Z}} b_{\alpha} \partial^{\alpha} \sum_{\mu \in \mathbf{Z}} b_{n, \mu} \partial^{\mu} \\
& =\sum_{\alpha \in \mathbf{Z}} \sum_{\mu \in \mathbf{Z}} \sum_{i=0}^{\infty}\left(\begin{array}{l}
\alpha \\
i
\end{array}\right) b_{\alpha} b_{n, \mu}^{(i)} \partial^{\alpha+\mu-i} \\
& =\sum_{v \in \mathbf{Z}}\left[\sum_{\alpha \in \mathbf{Z}} \sum_{i=0}^{\infty}\left(\begin{array}{l}
\alpha \\
i
\end{array}\right) b_{\alpha} b_{n, v-\alpha+i}^{(i)}\right] \partial^{v},
\end{aligned}
$$

we have

$$
\begin{aligned}
b_{n+1, v}= & \sum_{\alpha=0}^{\infty} \sum_{i=0}^{\alpha}\left(\begin{array}{l}
\alpha \\
i
\end{array}\right) b_{\alpha} b_{n, v-\alpha+i}^{(i)}+\sum_{\beta=1}^{\infty} \sum_{i=0}^{\infty}\left(\begin{array}{c}
-\beta \\
i
\end{array}\right) b_{-\beta} b_{n, v+\beta+i}^{(i)} \\
= & \sum_{\alpha=0}^{v+n} \sum_{i=0}^{\alpha}\left(\begin{array}{l}
\alpha \\
i
\end{array}\right) b_{\alpha} b_{n, v-\alpha+i}^{(i)} \\
& +\sum_{\alpha=v+n+1}^{\infty} \sum_{i=0}^{\alpha}\left(\begin{array}{l}
\alpha \\
i
\end{array}\right) b_{\alpha} b_{n, v-\alpha+i}^{(i)} \\
& +\sum_{l=1}^{\infty} \sum_{i=0}^{l-1}\left(\begin{array}{c}
i-l \\
i
\end{array}\right) b_{i-l} b_{n, v+l}^{(i)} .
\end{aligned}
$$

We now assume that $v \geqq-n-1$. In the first summation (3.3), since $0 \leqq \alpha \leqq v+n$ and $i \geqq 0$, we have $v-\alpha+i \geqq-n$. Hence by the induction hypothesis,

$$
\operatorname{val}_{i} b_{n, v-\alpha+i}^{(i)} \geqq \operatorname{val}_{t} b_{n, v-\alpha+i} \geqq J(v-\alpha+n)
$$

because the derivation $\partial$ does not decrease the valuation with respect to $t$. On the other hand, since $\alpha \geqq 0$, we know $\mathrm{val}_{t} b_{\alpha} \geqq J(\alpha+1)$. Therefore $\operatorname{val}_{t}\left(b_{\alpha} b_{n, v-\alpha+i}^{(i)}\right) \geqq J(\alpha+1)+J(v-\alpha+n) \geqq J(v+n+1)$, and hence

$$
\operatorname{val}_{t}\left(\sum_{\alpha=0}^{v+n} \sum_{i=0}^{\alpha}\left(\begin{array}{l}
\alpha \\
i
\end{array}\right) b_{\alpha} b_{n, v-\alpha+i}^{(i)}\right) \geqq J(v+n+1) .
$$

In the second summation (3.4), since $\alpha \geqq v+n+1$, we have

Therefore

$$
\operatorname{val}_{t}\left(\sum_{i=0}^{\alpha}\left(\begin{array}{l}
\alpha \\
i
\end{array}\right) b_{\alpha} b_{n, v-\alpha+i}^{(i)}\right) \geqq \operatorname{val}_{t} b_{\alpha} \geqq J(\alpha+1) \geqq J(v+n+2) .
$$

$$
\operatorname{val}_{t}\left(\sum_{\alpha=v+n+1}^{\infty} \sum_{i=0}^{\alpha}\left(\begin{array}{l}
\alpha \\
i
\end{array}\right) b_{\alpha} b_{n, v-\alpha+i}^{(i)}\right) \geqq J(v+n+1) .
$$

Finally, in the third summation (3.5), since $l \geqq 1$, we have $v+l \geqq v+1 \geqq-n$. Hence

$$
\operatorname{val}_{t}\left(\sum_{i=0}^{l-1}\left(\begin{array}{c}
i-\eta \\
i
\end{array}\right) b_{i-l} b_{n, v+l}^{(i)}\right) \geqq \operatorname{val}_{t} b_{n, v+l} \geqq J(v+l+n) \geqq J(v+n+1) .
$$


Therefore

$$
\operatorname{val}_{t}\left(\sum_{l=1}^{\infty} \sum_{i=0}^{l-1}\left(\begin{array}{c}
i-l \\
i
\end{array}\right) b_{i-l} b_{n, v+l}^{(i)}\right) \geqq J(v+n+1) .
$$

This completes the proof of the claim.

Because of this claim, $\sum_{n=0}^{\infty} b_{n, v} \in \mathscr{R}$ is a well-defined element. Therefore

$$
\sum_{n=0}^{\infty} Q^{n}=\sum_{n=0}^{\infty} \sum_{y \in \mathbf{Z}} b_{n, v} \partial^{v}=\sum_{v \in \mathbf{Z}}\left(\sum_{n=0}^{\infty} b_{n, v}\right) \partial^{v}
$$

Note that if $v \geqq 0$, then $\operatorname{val}_{t} \sum_{n=0}^{\infty} b_{n, v} \geqq J v$. This implies that $\sum_{n=0}^{\infty} Q^{n} \in \widehat{\mathscr{E}}$. But since $\left.\left(\sum_{n=0}^{\infty} Q^{n}\right)\right|_{\varepsilon=0} \in G$, it is an element of $\widehat{\mathscr{E}}^{\times}$. Since

$$
\begin{aligned}
1 & =\sum_{n=0}^{\infty}(1-P)^{n}-\sum_{n=1}^{\infty}(1-P)^{n} \\
& =\sum_{n=0}^{\infty}(1-P)^{n}-(1-P) \sum_{n=0}^{\infty}(1-P)^{n}=P \sum_{n=0}^{\infty}(1-P)^{n},
\end{aligned}
$$

the Neumann series gives $P^{-1} \in \hat{\mathscr{E}}^{\times}$. Thus we have established Lemma 3.1.

Note that $\hat{\mathscr{D}}^{\times}=\hat{\mathscr{E}}^{x} \cap \hat{\mathscr{D}}$ becomes a subgroup of $\hat{\mathscr{E}}^{\times}$. The inversion in $\hat{\mathscr{E}}^{\times}$ is given by the Neumann series. For example, $\left(1-t_{1} \partial\right)^{-1}=1+t_{1} \partial+t_{1}^{2} \partial^{2}$ $+t_{1}^{3} \partial^{3}+\ldots \in \hat{\mathscr{E}}^{\times}$. On the other hand,

$$
\begin{aligned}
(1-\partial)^{-1} & =\left(-\partial\left(1-\partial^{-1}\right)\right)^{-1}=-\partial^{-1}\left(1-\partial^{-1}\right)^{-1} \\
& =-\partial^{-1}\left(1+\partial^{-1}+\partial^{-2}+\partial^{-3}+\ldots\right)=-\partial^{-1}-\partial^{-2}-\partial^{-3}-\ldots \in \mathscr{E} .
\end{aligned}
$$

Therefore, we cannot evaluate $t$ by any numbers. In other words, the group $\hat{\mathscr{E}}^{\times}$is a purely formal object. However, as we have noted in Remark 1.5 , the use of group $\hat{\mathscr{E}}^{\times}$and its factorization gives actual convergence of the solutions of the KP system!

The algebra decomposition (1.7) was used effectively to determine all possible isospectral deformations of $L \in E$. The factorization theorem we are proving is its group version.

Theorem 3.2 (Generalized Birkhoff decomposition). For every element $U \in \hat{\mathscr{E}}^{\times}$, there exist a unique $S \in \mathscr{G}$ and a unique $Y \in \hat{\mathscr{D}}^{\times}$such that

$$
U=S^{-1} \cdot Y \text {. }
$$

Namely, we have a unique factorization

$$
\hat{\mathscr{E}}^{\mathrm{x}}=\mathscr{G} \cdot \hat{\mathscr{D}}^{\mathrm{x}}
$$


Proof. The uniqueness is trivial. Indeed, if $S_{1}^{-1} Y_{1}=S_{2}^{-1} Y_{2}$, then $S_{1} S_{2}^{-1}$ $=Y_{1} Y_{2}^{-1} \in \mathscr{G} \cap \hat{\mathscr{D}}^{\times}=\{1\}$. Hence $S_{1}=S_{2}$ and $Y_{1}=Y_{2}$. To show the existence of the decomposition, we have to solve the equation $S U \in \hat{\mathscr{D}}$ for an unknown $S \in \mathscr{G}$.

So let $U=\sum_{\beta \in \mathbf{Z}} u_{\beta} \partial^{\beta}$ and $S=1+\sum_{\alpha=1}^{\infty} s_{\alpha} \partial^{-\alpha}$. Then

$$
\begin{aligned}
S U & =\left(1+\sum_{\alpha=1}^{\infty} s_{\alpha} \partial^{-\alpha}\right)\left(\sum_{\beta \in \mathbf{Z}} u_{\beta} \partial^{\beta}\right) \\
& =\sum_{\beta \in \mathbf{Z}} u_{\beta} \partial^{\beta}+\sum_{\alpha=1}^{\infty} \sum_{\beta \in \mathbf{Z}} \sum_{i=0}^{\infty}\left(\begin{array}{c}
-\alpha \\
i
\end{array}\right) s_{\alpha} u_{\beta}^{(i)} \partial^{-\alpha+\beta-i} \\
& =\sum_{\beta \in \mathbf{Z}} u_{\beta} \partial^{\beta}+\sum_{\gamma \in \mathbf{Z}}\left(\sum_{\alpha=1}^{\infty} \sum_{i=0}^{\infty}\left(\begin{array}{c}
-\alpha \\
i
\end{array}\right) s_{\alpha} u_{\gamma+\alpha+i}^{(i)}\right) \partial^{\gamma} .
\end{aligned}
$$

Therefore the equation we have to solve is a system of algebraic equations

$$
u_{-\beta}+\sum_{\alpha=1}^{\infty} \sum_{i=0}^{\infty}\left(\begin{array}{c}
-\alpha \\
i
\end{array}\right) s_{\alpha} u_{\alpha-\beta+i}^{(i)}=0 \quad \text { for } \beta=1,2,3, \ldots
$$

Define

$$
\begin{aligned}
& \mathbf{u}=\left(u_{-1}, u_{-2}, u_{-3}, \ldots\right), \\
& \mathbf{s}=\left(s_{1}, s_{2}, s_{3}, \ldots\right)
\end{aligned}
$$

and

$$
M=\left[\sum_{i=0}^{\infty}\left(\begin{array}{c}
-\alpha \\
i
\end{array}\right) u_{\alpha-\beta+i}^{(i)}\right]_{\alpha, \beta=1,2,3, \ldots}
$$

where $M$ is a square matrix of infinite size with coefficients in $\mathscr{R}$. Equation (3.8) now reads

$$
\mathbf{s} M=-\mathbf{u} .
$$

Therefore the solution $\mathbf{s}$ is given by $\mathbf{s}=-\mathbf{u} M^{-1}$. Our idea is to define $M^{-1}$ by the Neumann series $\sum_{n=0}^{\infty}(1-M)^{n}$, and use a similar technique developed in the proof of Lemma 3.1 to establish well-definedness of $\sum_{n=0}^{\infty}(1-M)^{n}$ and $\mathbf{u} \cdot M^{-1}$. Since $\mathbf{s}$ determines all the coefficients of $S$, well-definedness of $\mathbf{u} \cdot \sum_{n=0}^{\infty}(1-M)^{n}$ implies the existence of $S$ such that $S U \in \hat{\mathscr{D}}$.

Let

and

$$
\begin{aligned}
N & =1-M=\left[a_{\mu v}\right]_{\mu, v=1,2,3, \ldots} \\
N^{n} & =\left[a_{n, \mu v}\right]_{\mu, v}=1,2,3, \ldots
\end{aligned}
$$


Since $a_{\mu \nu}=\delta_{\mu \nu}-\sum_{i=0}^{\infty}\left(\begin{array}{c}-\mu \\ i\end{array}\right) u_{\mu-\nu+i}^{(i)}$, we have

$$
\left.a_{\mu \mu}\right|_{t=0}=1-\left.\sum_{i=0}^{\infty}\left(\begin{array}{c}
-\mu \\
i
\end{array}\right) u_{i}^{(i)}\right|_{t=0}=-\left.\sum_{i=1}^{\infty}\left(\begin{array}{c}
-\mu \\
i
\end{array}\right) u_{i}^{(i)}\right|_{t=0}=0
$$

because $\left.u_{i}\right|_{t=0}=0$ for all $i>0$. Similarly, if $\mu>v$, then

$$
\left.a_{\mu v}\right|_{t=0}=-\left.\sum_{i=0}^{\infty}\left(\begin{array}{c}
-\mu \\
i
\end{array}\right) u_{\mu-v+i}^{(i)}\right|_{t=0}=0
$$

Because of the growth order condition for $u_{v}$ 's, we can find a positive real number $J$ such that

as before.

$$
\operatorname{val}_{t} a_{\mu \nu} \geqq J(\mu-v+1) \quad \text { for all } \mu-v \geqq-1
$$

Claim. For every $n \geqq 1$ we have

$$
\operatorname{val}_{t} a_{n, \mu \nu} \geqq J(\mu-v+n) \quad \text { if } \mu-v \geqq-n .
$$

Proof of the Claim. If $n=1$, then $a_{1, \mu v}=a_{\mu \nu}$ and (3.11) follows from (3.10). Assume that (3.11) holds for some $n \geqq 1$. Since $N^{n+1}=N^{n} \cdot N$,

$$
\begin{aligned}
a_{n+1, \mu \nu} & =\sum_{l=1}^{\infty} a_{n, \mu l} a_{l v} \\
& =\sum_{l=1}^{v-1} a_{n, \mu l} a_{l v}+\sum_{l=v}^{\mu+n} a_{n, \mu l} a_{l v}+\sum_{l=\mu+n+1}^{\infty} a_{n, \mu l} a_{l v} .
\end{aligned}
$$

Let us assume that $\mu-v \geqq-n-1$. hence

In the first term of $(3.12)$, since $v-1 \geqq l$, we have $\mu-l \geqq \mu-v+1 \geqq-n$, and

$$
\operatorname{val}_{t}\left(a_{n, \mu l} a_{l v}\right) \geqq \operatorname{val}_{t} a_{n, \mu l} \geqq J(\mu-l+n) \geqq J(\mu-v+n+1) .
$$

Therefore

$$
\operatorname{val}_{t}\left(\sum_{l=1}^{v-1} a_{n, \mu l} a_{l v}\right) \geqq J(\mu-v+n+1) .
$$

In the second term of (3.12), since $v \leqq l \leqq \mu+n$, we have $\mu-l \geqq-n$ and $l-v \geqq 0$. Hence

$$
\operatorname{val}_{t}\left(a_{n, \mu l} a_{l v}\right)=\operatorname{val}_{t} a_{n, \mu l}+\operatorname{val}_{t} a_{l v} \geqq J(\mu-l+n)+J(l-v+1)=J(\mu-v+n+1) .
$$

Therefore

$$
\operatorname{val}_{t}\left(\sum_{l=v}^{\mu+n} a_{n, \mu l} a_{l v}\right) \geqq J(\mu-v+n+1) .
$$


Finally, in the third term of (3.12), $l \geqq \mu+n+1$ implies $l-v \geqq \mu-v+n+1$. Therefore

$$
\operatorname{val}_{t}\left(\sum_{i=\mu+n+1}^{\infty} a_{n, \mu l} a_{l v}\right) \geqq \operatorname{val}_{t} a_{l v} \geqq J(l-v+1) \geqq J(\mu-v+n+1)
$$

This completes the proof of the claim.

By this claim we can conclude that $\sum_{n=0}^{\infty} a_{n, \mu \nu} \in \mathscr{R}$ is well-defined for all $\mu, v$ $=1,2,3, \ldots$ Therefore

$$
M^{-1}=\sum_{n=0}^{\infty}(1-M)^{n}=\left[\sum_{n=0}^{\infty} a_{n, \mu \nu}\right]_{\mu, v}
$$

is well-defined. Let $M^{-1}=\left[b_{\mu \nu}\right]_{\mu, v=1,2,3, \ldots}$, namely $b_{\mu v}=\sum_{n=0}^{\infty} a_{n, \mu \nu}$. If $\mu>v$, then $\operatorname{val}_{t} b_{\mu v}=\operatorname{val}_{t} \sum_{n=0}^{\infty} a_{n, \mu v} \geqq J(\mu-v)$. Therefore

$$
s_{v}=-\sum_{\mu=1}^{\infty} u_{-\mu} b_{\mu \nu}=-\sum_{\mu=1}^{v} u_{-\mu} b_{\mu v}-\sum_{\mu=v+1}^{\infty} u_{-\mu} b_{\mu v}
$$

is a well-defined element in $\mathscr{R}$. Thus we have established the existence of $S \in \mathscr{G}$ such that $S U \in \mathscr{G}$.

Finally, let $Y=S U$. Then $Y \in \hat{\mathscr{D}}^{\times}$and $U=S^{-1} \cdot Y$. This completes the proof of Theorem 3.2.

When the differential algebra $(R, \partial)$ is trivial, i.e. if $\partial: R \rightarrow R$ is the zero-map, we write $\partial=\lambda$ and consider it as a commuting variable. Then $\hat{\mathscr{E}}$ can be identified with a formal loop space of $\mathscr{R}=R \| t \rrbracket$ with pointwise multiplication. If $\lambda$ is a complex variable, then $P=\sum_{v \in \mathbf{Z}} a_{v} \lambda^{v} \in \widehat{E}$ is a map of the unit circle in $\mathbb{C}$ into $\mathscr{R}$. The group $\hat{\mathscr{E}}^{\times}$is the connected component of the identity operator 1 in the group of all invertible elements of $\hat{E}$. The subgroup $\mathscr{G}$ consists of a function $S: S^{1} \ni \lambda \mapsto S(\lambda) \in \mathscr{R}$ which is holomorphic on the upper hemisphere of $P_{\mathbf{c}}^{1}$ with $S(\infty)=1$. The other subgroup $\hat{\mathscr{D}}^{\times}$of $\hat{\mathscr{E}}^{\times}$consists of a function $Y: S^{1} \ni \lambda \mapsto Y(\lambda) \in \mathscr{R}$ which is holomorphic on the lower hemisphere of $P_{\mathbf{C}}^{1}$ such that $Y(0)$ is invertible in $\mathscr{R}$.

Thus we recover the usual Birkhoff decomposition of the connected component of the identity of a loop group (Pressley-Segal [10]).

In the rest of this section, notations coincide with Sect. 2 , namely $\widehat{\mathscr{E}}^{\times}, \hat{\mathscr{E}}_{0}^{\times}$ etc. are sets of super micro-differential operators.

Lemma 3.3. (a) Every element $P \in \widehat{\mathscr{E}}^{\times}$has an inverse in $\hat{\mathscr{E}}^{\times}$.

(b) If $P \in \mathscr{E}_{0}^{\times}$, then $P^{-1} \in \widehat{\mathscr{E}}_{0}^{\times}$.

Proof. The first part (a) can be proved by the same argument used in the proof of Lemma 3.1. The only necessary alternation is to replace the Leibnitz rule (1.3) by its super analogue (2.7), otherwise the proof is identical.

To establish (b), let $P=\sum_{v \in \mathbf{Z}} a_{v} \theta^{v} \in \hat{\mathscr{E}}_{0}^{\times}, Q=1-P=\sum_{v \in \mathbf{Z}} b_{v} \theta^{v}$ and $Q^{n}=\sum_{\nu \in \mathbf{Z}} b_{n, v} \theta^{v}$. 
Since

$$
P^{-1}=\sum_{n=0}^{\infty}(1-P)^{n}=\sum_{n=0}^{\infty} Q^{n}=1+\sum_{n=1}^{\infty} \sum_{v \in \mathbf{Z}} b_{n, v} \theta^{v}=1+\sum_{v \in \mathbf{Z}}\left(\sum_{n=1}^{\infty} b_{n, v}\right) \theta^{v}
$$

we have to show that $\left(\sum_{n=1}^{\infty} \widetilde{b_{n, v}}\right)=\tilde{v}$. Therefore we need to prove that $\widetilde{b_{n, v}}=\tilde{v}$ for all $n \geqq 1$.

If $n=1$, then $\widetilde{b_{1, v}}=\widetilde{b_{v}}=\left(\widetilde{\delta_{v 0}-a_{v}}\right)=\widetilde{a_{v}}=\tilde{v}$. So assume that $\widetilde{b_{n, v}}=\tilde{v}$ for some $n \geqq 1$. Now, since

$$
\begin{aligned}
Q^{n+1}=Q Q^{n} & =\sum_{v \in \mathbf{Z}} b_{v} \theta^{v} \sum_{\mu \in \mathbf{Z}} b_{n, \mu} \theta^{\mu} \\
& =\sum_{v \in \mathbf{Z}} \sum_{\mu \in \mathbf{Z}} \sum_{i=0}^{\infty}\left[\begin{array}{l}
v \\
i
\end{array}\right](-1)^{b_{n, \mu}(v-i)} b_{v} b_{n, \mu}^{[i]} \theta^{v+\mu-i} \\
& =\sum_{\alpha \in \mathbf{Z}}\left(\sum_{v \in \mathbf{Z}} \sum_{i=0}^{\infty}\left[\begin{array}{l}
v \\
i
\end{array}\right](-1)^{(\alpha+i-v)(\nu-i)} b_{v} b_{n, \alpha+i-v}^{[i]}\right) \theta^{\alpha},
\end{aligned}
$$

we have

$$
b_{n+1, \alpha}=\sum_{v \in Z} \sum_{i=0}^{\infty}\left[\begin{array}{l}
v \\
i
\end{array}\right](-1)^{(\alpha+i-v)(v-i)} b_{v} b_{n, a+i-v}^{[i]} .
$$

Note here that if $\tilde{b}=\tilde{n}$, then $\widetilde{b^{[i j}}=(\widetilde{n+i})$ because $\theta$ is an odd derivation. Thus we have

$$
\begin{aligned}
\widetilde{\left(b_{v} b_{n, \alpha-v+i}^{[i]}\right)} & =\tilde{b}_{v}+\overparen{b_{n, \alpha-v+i}^{[i]}} \\
& =\tilde{v}+\tilde{\alpha}-\tilde{v}+\tilde{i}+\tilde{i}=\tilde{\alpha} .
\end{aligned}
$$

Therefore $\tilde{b}_{n+1, \alpha}=\tilde{\alpha}$, namely, $P^{-1} \in \hat{\mathscr{E}}_{0}^{x}$. This completes the proof.

The following factorization is the main tool to establish the solvability of the super KP system.

Theorem 3.4 (Super analogue of generalized Birkhoff decomposition). Let $\hat{\mathscr{E}}^{\times}$, $\hat{\mathscr{E}}_{0}^{\times}, \hat{\mathscr{D}}^{\times}, \mathscr{G}, \hat{\mathscr{D}}_{0}^{\times}$and $\mathscr{G}_{0}$ be the groups of super micro-differential operators defined in Sect. 2. Then for every $U \in \hat{\mathscr{E}}^{\times}$, there is a unique element $S$ of $\mathscr{G}$ and a unique element $Y$ of $\hat{D}^{\times}$such that

$$
U=S^{-1} \cdot Y
$$

Moreover, if $U$ is an even operator in $\hat{\mathscr{E}}_{0}^{\times}$, then the above $S$ and $Y$ are both even;

$$
S \in \mathscr{G}_{0} \text { and } Y \in \hat{\mathscr{D}}_{0}^{\times} .
$$

In other words, we have a unique factorization

$$
\hat{\mathscr{E}}^{\times}=\mathscr{G} \cdot \hat{\mathscr{D}}^{\times} \quad \text { and } \quad \hat{\mathscr{E}}_{0}^{\times}=\mathscr{G}_{0} \cdot \hat{\mathscr{D}}_{0}^{\times}
$$


Proof. The proof is again the same as that of Theorem 3.2. We start with $U$ $=\sum_{v \in \mathbf{Z}} u_{v} \theta^{v}$ and let $S=1+\sum_{\alpha=1}^{\infty} S_{\alpha} \theta^{\alpha}$. The equation we want to solve is $S U \in \hat{\mathscr{D}}$.

By a similar calculation, we obtain

$$
u_{-\nu}+\sum_{\mu=1}^{\infty} \sum_{i=0}^{\infty}\left[\begin{array}{c}
-\mu \\
i
\end{array}\right](-1)^{u_{\mu-\nu+i}(\mu+i)} s_{\mu} u_{\mu-v+i}^{[i]}=0 .
$$

So let

and

$$
\begin{aligned}
M & =\left[\sum_{i=0}^{\infty}\left[\begin{array}{c}
-\mu \\
i
\end{array}\right](-1)^{\tilde{\mu}_{\mu-\nu+i}(\mu+i)} u_{\mu-\nu+i}^{[i]}\right]_{\mu, v=1,2,3, \ldots}, \\
N & =1-M=\left[a_{\mu \nu}\right]_{\mu, v=1,2,3, \ldots} \\
N^{n} & =\left[a_{n, \mu \nu}\right]_{\mu, v}=1,2,3, \ldots .
\end{aligned}
$$

Then the well-definedness of $\sum_{n=0}^{\infty}(1-M)^{n}$ and the product

$$
\left(u_{-1}, u_{-2}, u_{-3}, \ldots\right) \sum_{n=0}^{\infty}(1-M)^{n}
$$

can be shown in the same way. Therefore,

$$
\left(s_{1}, s_{2}, s_{3}, \ldots\right)=-\left(u_{-1}, u_{-2}, u_{-3}, \ldots\right) M^{-1}
$$

gives a solution. Letting $Y=S U$, we obtain (3.13).

When $U$ is an even operator, then $\tilde{u}_{v}=\tilde{v}$. Since

$$
a_{\mu \nu}=\delta_{\mu \nu}-\sum_{i=0}^{\infty}\left[\begin{array}{c}
-\mu \\
i
\end{array}\right](-1)^{\tilde{\mu}_{\mu-\nu+i}(\mu+i)} u_{\mu-\nu+1}^{[i]},
$$

we have $\widetilde{a_{\mu \nu}}=\widetilde{u_{\mu-v+i}^{(i)}}=(\widetilde{\mu-v})$. Now if $\tilde{a}_{n, \mu \nu}=(\widetilde{\mu-v})$, then

$$
\tilde{a}_{n+1, \mu v}=\left(\sum_{l=1}^{\infty} \widetilde{a_{n, \mu l} a_{l v}}\right)=\widetilde{a_{n, \mu l}}+\widetilde{a_{l v}}=\tilde{\mu}-\tau+\tau-\tilde{v}=(\widetilde{\mu-v}) .
$$

Therefore $\quad \tilde{a}_{n, \mu \nu}=(\widetilde{\mu-\nu})$ for $\quad$ all $\quad n=1,2,3, \ldots$ Let $\quad M^{-1}=\sum_{n=0}^{\infty}(1-M)^{n}$ $=\left[b_{\mu \nu}\right]_{\mu, v=1,2,3, \ldots .}$. Then

Therefore

$$
\widetilde{b_{\mu \nu}}=\left(\sum_{n=0}^{\infty} \tilde{a}_{n, \mu \nu}\right)=(\widetilde{\mu-v})
$$

$$
\tilde{s}_{v}=-\left(\sum_{\mu=1}^{\infty} \widetilde{u_{-\mu} b_{\mu v}}\right)=-\tilde{\mu}+\tilde{\mu}-\tilde{v}=\tilde{v}
$$


namely, $S \in \mathscr{G}_{0}$. Since $U$ and $S$ are both even operators, we have $Y=S U \in \hat{\mathscr{D}}_{0}^{\times}$, thus (3.14) is obtained.

The uniqueness of the decomposition is trivial since $\mathscr{G} \cap \hat{\mathscr{D}}^{\times}=\mathscr{G}_{0} \cap \hat{\mathscr{D}}_{0}^{\times}=\{1\}$. This completes the proof of Theorem 3.4.

\section{Some linear total differential equations}

To establish the uniqueness of the Cauchy problem for the generalized KP system (1.19), we used the following fact; every solution $S$ of (1.19) gives rise to a solution $U$ of the universal equation (1.29). In Sect. 1 we proved this fact by assuming that Eq. (1.26) $d Y=Z_{+} Y$ has a unique solution in $\hat{\mathscr{D}}^{\times}$. Recall that if $S$ is a solution of (1.19), then $Z_{+}$satisfies the intergrability condition $d Z_{+}=Z_{+} \wedge Z_{+}$.

For a finite dimensional situation, solvability of (1.26) under the integrability condition is a well-known fact. But in our case, since the group we use and the base space are both of infinite dimension, we need some technical tricks to establish this fact.

Let $D, \mathscr{D}$ and $\hat{\mathscr{D}}$ be algebras of ordinary differential operators introduced in Sect. 1.

Theorem 4.1. Let $\omega=\sum_{n=1}^{\infty} P_{n} d t_{n}$ be a $\mathscr{D}$-valued 1-form satisfying

(a) there is a positive real number $c>0$ such that

$$
\text { ord } P_{n} \leqq \frac{n}{c} \quad \text { for all } n \geqq 1
$$

(b) $\omega$ is integrable, i.e. $d \omega=\omega \wedge \omega$.

Then for every given operator $Y(0) \in D$, there is a unique solution $Y \in \hat{\mathscr{D}}$ of the linear total differential equation

$$
d Y=\omega Y
$$

having $Y(0)$ as its initial value; $\left.Y\right|_{t=0}=Y(0)$.

Proof. Note that (4.1) is equivalent to the system

$$
\frac{\partial Y}{\partial t_{n}}=P_{n} \cdot Y
$$

Let $Y=\sum_{l=0}^{\infty} y_{l} \partial^{l}$. Then the zero-th order term of (4.2) gives an equation

$$
\frac{\partial y_{0}}{\partial t_{n}}=P_{n}\left(y_{0}\right)
$$

where $P_{n}\left(y_{0}\right)$ means the function obtained by applying the differential operator $P_{n}$ to the function $y_{0}$. Therefore, we have to establish, first of all, the following. 
Lemma 4.2. Let $\omega$ be as in the theorem. Then for every $f \in R$, there is a unique solution $y \in \mathscr{R}$ of the system

such that $\left.y\right|_{t=0}=f \in R$.

$$
\frac{\partial y}{\partial t_{n}}=P_{n}(y), \quad n=1,2,3, \ldots
$$

Proof of the Lemma. For an individual $t_{n}$, it is easy to solve $\partial y / \partial t_{n}=P_{n}(y)$. Actually, let

$$
y=\sum_{i=0}^{\infty} \frac{b_{i}}{i !} t_{n}^{i}, b_{i} \in R, \quad P_{n}=\sum_{k=0}^{n / c} a_{k} \partial^{k}
$$

and

Then

$$
a_{k}=\sum_{j=0}^{\infty} a_{k, j} \frac{t_{n}^{j}}{j !}, \quad a_{k, j} \in R
$$

$$
\begin{aligned}
0 & =\frac{\partial y}{\partial t_{n}}-P_{n}(y)=\frac{\partial y}{\partial t_{n}}-\sum_{k \geqq 0} a_{k} y^{(k)} \\
& =\sum_{i \geqq 0} b_{i} \frac{t_{n}^{i-1}}{(i-1) !}-\sum_{k \geqq 0} \sum_{j=0}^{\infty} \sum_{i=0}^{\infty} a_{k, j} b_{i}^{(k)} \frac{1}{i ! j !} t_{n}^{i+j} \\
& =\sum_{\alpha=0}^{\infty}\left[b_{\alpha+1}-\sum_{i=0}^{\alpha} \sum_{k \geqq 0}\left(\begin{array}{l}
\alpha \\
i
\end{array}\right) a_{k, \alpha-i} b_{i}^{(k)}\right] \frac{t_{n}^{\alpha}}{\alpha !},
\end{aligned}
$$

namely we obtain

$$
b_{\alpha+1}=\sum_{i=0}^{\alpha} \sum_{k \geqq 0}\left(\begin{array}{l}
\alpha \\
i
\end{array}\right) a_{k, \alpha-i} b_{i}^{(k)}
$$

Therefore $y$ is uniquely determined by $b_{0}=\left.y\right|_{t_{n}=0}$.

We can repeat the above procedure for $t_{1}, t_{2}, t_{3}, \ldots$ The integrability condition (b) establishes the compatibility of $t_{n}$-dependence of $y$. In this way we obtain a well-defined function $y \in \mathscr{R}$.

Now let us go back to the proof of the theorem. If the differential algebra $(R, \partial)$ is trivial, then $Y$ and $P_{n}$ 's are all multiplication operators. Therefore, the above Lemma establishes the existence of a formal solution $Y$. (We will show later that it is actually an element of $\hat{\mathscr{D}}$.)

So let us assume that $(R, \partial)$ is non-trivial. Now let $x$ be a dual operator of $\partial$. Namely, $x$ satisfies

$$
[R, x]=0 \text { and }[\partial, x]=1 .
$$

(Of course $x$ may not be an element of $R$.) The reason why we want to use such an operator $x$ is because its introduction makes computation much easier. Logically, we do not need $x$.

In this proof we use the following simplified notation;

$$
A \cdot[, B]^{n}=[[\ldots[[A, \underbrace{B], B], \ldots, B], B]}_{n \text {-times }}] .
$$

Namely $A \cdot[, B]=[A, B], A \cdot[, B]^{2}=[[A, B], B]$ etc. 
Claim 1. The equation $\partial Y / \partial t_{n}=P_{n} Y$ is equivalent to

$$
\frac{\partial}{\partial t_{n}}\left(\sum_{i=0}^{l} \frac{x^{i}}{i !} y_{l-i}\right)=P_{n}\left(\sum_{i=0}^{l} \frac{x^{i}}{i !} y_{l-i}\right) \text { for all } l \geqq 0 \text {. }
$$

Let's observe that the above claim establishes the unique existence of a formal solution $Y$. Indeed, assume that we know $y_{0}, y_{1}, \ldots, y_{l-1}$. Lemma 4.2 says that Eq. (4.6) is solvable for every initial data. Therefore, if we give $y_{l} l_{t=0}$, (4.6) determines $y_{l} \in \mathscr{R}$. If we continue this procedure, then we obtain all $y_{0}, y_{1}, y_{2}, \ldots$ Thus we get a formal solution $\sum_{i=0}^{\infty} y_{l} \partial^{l}=Y$ of (4.2). Again its compatibility of $t$-dependence follows from (b).

Proof of Claim 1. We need the following formulas. Let $P_{n}=\sum_{j \geqq 0} a_{n, j} \partial^{j}$. Then

$$
\begin{gathered}
\sum_{j \geqq 0}\left(\begin{array}{l}
j \\
l
\end{array}\right) a_{n, j} \partial^{j-l}=P_{n} \cdot \frac{[, x]^{l}}{l !}, \\
P_{n} \cdot \frac{x^{j}}{j !}=\sum_{k=0}^{j} \frac{x^{j-k}}{(j-k) !} P_{n} \cdot \frac{[, x]^{k}}{k !} .
\end{gathered}
$$

Since

$$
\begin{aligned}
0 & =\frac{\partial Y}{\partial t_{n}}-P_{n} Y=\sum_{l=0}^{\infty} \frac{\partial y_{l}}{\partial t_{n}} \partial^{l}-\sum_{j \geqq 0} a_{n, j} \partial^{j} \sum_{k=0}^{\infty} y_{k} \partial^{k} \\
& =\sum_{l=0}^{\infty} \frac{\partial y_{l}}{\partial t_{n}} \partial^{l}-\sum_{j \geqq 0} \sum_{k=0}^{\infty} \sum_{i=0}^{j}\left(\begin{array}{l}
j \\
i
\end{array}\right) a_{n, j} y_{k}^{(i)} \partial^{j+k-i} \\
& =\sum_{l=0}^{\infty}\left(\frac{\partial y_{l}}{\partial t_{n}}-\sum_{j \geqq 0} \sum_{k=0}^{\infty}\left(\begin{array}{c}
j \\
j+k-l
\end{array}\right) a_{n, j} y_{k}^{(j+k-l)}\right) \partial^{l},
\end{aligned}
$$

we have

$$
\begin{aligned}
\frac{\partial y_{l}}{\partial t_{n}} & =\sum_{j \geqq 0} \sum_{k=0}^{\infty}\left(\begin{array}{c}
j \\
l-k
\end{array}\right) a_{n, j} y_{k}^{(j+k-l)}=\sum_{j \geqq 0} \sum_{k=0}^{l}\left(\begin{array}{l}
j \\
k
\end{array}\right) a_{n, j} y_{l-k}^{(j-k)} \\
& =\sum_{k=0}^{l}\left(\sum_{j \geqq 0}\left(\begin{array}{l}
j \\
k
\end{array}\right) a_{n, j} \partial^{j-k}\right)\left(y_{l-k}\right)=\sum_{k=0}^{l}\left(P_{n} \cdot \frac{[, x]^{k}}{k !}\right)\left(y_{l-k}\right) .
\end{aligned}
$$

Therefore,

$$
\begin{aligned}
\frac{\partial}{\partial t_{n}}\left(\sum_{i=0}^{l} \frac{x^{i}}{i !} y_{l-i}\right) & =\sum_{i=0}^{l} \frac{x^{i}}{i !} \frac{\partial y_{l-i}}{\partial t_{n}}=\sum_{i=0}^{l} \sum_{k=0}^{l-i} \frac{x^{i}}{i !}\left(P_{n} \cdot \frac{[, x]^{k}}{k !}\right)\left(y_{l-i-k}\right) \\
& =\sum_{j=0}^{l} \sum_{k=0}^{j} \frac{x^{j-k}}{(j-k) !}\left(P_{n} \cdot \frac{[, x]^{k}}{k !}\right)\left(y_{l-j}\right) \\
& =\sum_{j=0}^{l} P_{n}\left(\frac{x^{j}}{j !} y_{l-j}\right)=P_{n}\left(\sum_{i=0}^{l} \frac{x^{i}}{i !} y_{l-i}\right) .
\end{aligned}
$$


Proof of (4.7). Since

we have

$$
\left[P_{n}, x\right]=\sum_{j \geqq 0} a_{n, j}\left[\partial^{j}, x\right]=\sum_{j \geqq 0} j a_{n, j} \partial^{j-1}
$$

$$
\begin{aligned}
P_{n} \cdot[, x]^{l} & =\sum_{j \geqq 0} j(j-1)(j-2) \ldots(j-l+1) a_{n, j} \partial^{j-l} \\
& =l ! \sum_{j \geqq 0}\left(\begin{array}{l}
j \\
l
\end{array}\right) a_{n, j} \partial^{j-l} .
\end{aligned}
$$

Proof. of (4.8). If $j=0$, then (4.8) simply says that $P_{n}=P_{n}$. So assume that $(4.8)$ is true for some $j$. Then

$$
\begin{aligned}
P_{n} \cdot \frac{x^{j}+1}{(j+1) !}= & P_{n} \cdot\left(\frac{x^{j}}{j !} \cdot \frac{x}{j+1}\right)=\frac{1}{j+1}\left[P_{n} \cdot \frac{x^{j}}{j !}, x\right]+\frac{x}{j+1} P_{n} \cdot \frac{x^{j}}{j !} \\
= & \frac{1}{j+1}\left[\sum_{k=0}^{j} \frac{x^{j-k}}{(j-k) !} P_{n} \cdot \frac{[, x]^{k}}{k !}, x\right]+\frac{x}{j+1} \sum_{k=0}^{j} \frac{x^{j-k}}{(j-k) !} P_{n} \cdot \frac{[, x]^{k}}{k !} \\
= & \frac{1}{j+1} \sum_{k=0}^{j} \frac{x^{j-k}}{(j-k) !} \cdot \frac{(k+1) !}{k !} \cdot P_{n} \cdot \frac{[, x]^{k+1}}{(k+1) !} \\
& +\frac{1}{j+1} \sum_{k=0}^{j} \frac{x^{j-k+1}}{(j-k+1) !} \frac{(j-k+1) !}{(j-k) !} P_{n} \cdot \frac{[, x]^{k}}{k !} \\
= & \frac{1}{j+1} \sum_{k=1}^{j+1} \frac{x^{j-k+1}}{(j-k+1) !} k P_{n} \cdot \frac{[, x]^{k}}{k !} \\
& +\frac{1}{j+1} \sum_{k=0}^{j} \frac{x^{j-k+1}}{(j-k+1) !}(j-k+1) P_{n} \cdot \frac{[, x]^{k}}{k !} \\
= & \sum_{k=0}^{j+1} \frac{x^{j-k+1}}{(j-k+1) !} \cdot \frac{k+j-k+1}{j+1} P_{n} \cdot \frac{[, x]^{k}}{k !} \\
= & \sum_{k=0}^{j+1} \frac{x^{j-k+1}}{(j-k+1) !} P_{n} \cdot \frac{[, x]^{k}}{k !} .
\end{aligned}
$$

Thus induction on $j$ works to establish (4.8).

Finally we have to establish a growth order condition to show that $Y$ $=\sum_{l=0}^{\infty} y_{l} \partial^{l}$ is an well-defined element of $\hat{\mathscr{D}}$. Let $N=\operatorname{ord}(Y(0))$. Note that by assumption (a), we have ord $P_{n} \leqq \frac{n}{c}$. Therefore, by $(4.7)$, ord $\left(P_{n} \cdot\left([, x]^{k}\right) / k !\right) \leqq \frac{n}{c}-k$. Namely, $P_{n} \cdot[, x]^{k}=0$ if $k>\frac{n}{c}$. Hence in (4.9), we have

$$
\frac{\partial y_{l}}{\partial t_{n}}=\sum_{k=0}^{l}\left(P_{n} \cdot \frac{[, x]^{l-k}}{(l-k) !}\right)\left(y_{k}\right)=\sum_{k \geqq l-\frac{n}{c}}^{l}\left(P_{n} \cdot \frac{[, x]^{l-k}}{(l-k) !}\right)\left(y_{k}\right)
$$


Since $\left.Y\right|_{t=0}=Y(0) \in D$ is of order $N$ and $\left.y_{l}^{(i)}\right|_{t=0}=\left(\left.y_{t}\right|_{t=0}\right)^{(i)}$, we have

$$
\left.\frac{\partial y_{l}}{\partial t_{n}}\right|_{t=0}=\sum_{j \geqq N+1}^{l}\left(P_{n \mid t=0} \cdot \frac{[, x]^{l-j}}{(l-j) !}\right)\left(\left.y_{j}\right|_{t=0}\right)=0
$$

for all $l \geqq \frac{n}{c}+N+1$.

Let $J=\left(n_{1}, n_{2}, \ldots, n_{r}\right)$ be an $r$-tuple of positive integers and let $|J|=n_{1}+n_{2}$ $+\ldots+n_{r}$. We denote

Claim 2.

$$
\partial_{J}=\prod_{j \in J} \frac{\partial}{\partial t_{j}}=\frac{\partial^{r}}{\partial t_{n_{1}} \partial t_{n_{2}} \ldots \partial t_{n_{r}}} .
$$

$$
\left.\partial_{J} y_{t}\right|_{t=0}=0 \quad \text { for all } l>\frac{1}{c}|J|+N+1 .
$$

Proof of Claim 2. We use induction on $r$. If $r=1$, then it reduces to (4.10). Assume that (4.12) is true for all $s \leqq r$. For every sub-tuple $I \subset J$, we define $I^{c}=$ complement of $I$ in $J$ and denote $\bar{\partial}_{I}=\prod_{i \in I} \partial / \partial t_{i}$. By the induction hypothesis, $\left.\partial_{I} y_{l}\right|_{t=0}=0$ for all $l>\frac{1}{c}|J|+N+1$. Now assume that $l>\frac{1}{c}|J|+N+1+\frac{n}{c}$. Then

$$
\begin{aligned}
\left.\frac{\partial}{\partial t_{n}} \partial_{J} y_{l}\right|_{t=0} & =\left.\partial_{J}\left(\sum_{k \geqq l-\frac{n}{c}}^{l}\left(P_{n} \cdot \frac{[, x]^{l-k}}{(l-k) !}\right)\left(y_{k}\right)\right)\right|_{t=0} \\
& =\sum_{I \in J} \sum_{k \geqq l-\frac{n}{c}}^{l}\left(\left.\left(\partial_{I c} P_{n}\right)\right|_{t=0} \cdot \frac{[, x]^{l-k}}{(l-k) !}\right)\left(\left.\left(\partial_{I} y_{k}\right)\right|_{t=0}\right)=0
\end{aligned}
$$

since $k>\frac{1}{c}|J|+N+1$. This completes the proof of Claim 2 .

Therefore, we have

$$
\operatorname{val}_{t} y_{l} \geqq n_{1}+n_{2}+\ldots+n_{r}
$$

for all $l>\frac{1}{c}\left(n_{1}+n_{2}+\ldots+n_{r}\right)+N+1$. If we define $M_{Y}=N+1$ and $N_{Y}=$ any integer greater than $c(N+1)$, then the above inequality implies that

$$
\text { val }_{t} y_{l}>c l-N_{Y} \quad \text { for all } l>M_{Y} \text {. }
$$

Therefore, $Y=\sum_{l=0}^{\infty} y_{l} \partial^{l}$ is a well-defined element of $\hat{\mathscr{D}}$. This completes the proof of Theorem 4.1 .

Lemma 4.3. Let $\Omega=\sum_{n=1}^{\infty} d t_{n} \partial^{n}$. If the universal equation (1.29) $d U=\Omega U$ has two solutions $U$ and $V \in \hat{\mathscr{E}}$ with the same initial value $\left.U\right|_{t=0}=\left.V\right|_{t=0}$, then $U=V$. 
Proof. Let $W=U-V$. Note that $\left.W\right|_{t=0}=\left.U\right|_{t=0}-\left.V\right|_{t=0}=0$. Since (1.29) is linear, we have $d W=\Omega W$, namely, $\partial W / \partial t_{n}=\partial^{n} \cdot W$. Therefore, $\partial_{J} W=\partial^{|S|} W$, where $\partial_{J}$ is the operator defined in (4.11). Thus $\left.\left(\partial_{J} W\right)\right|_{t=0}=\left.\partial^{|J|} W\right|_{t=0}=0$ for all $J$ $=\left(n_{1}, n_{2}, \ldots, n_{r}\right)$. This means that $W$ does not depend on $t$. Since $\left.W\right|_{t=0}=0$, we can conclude that $W=0$. This completes the proof of Lemma 4.3.

A similar statement is valid for the super case.

Lemma 4.4. Let

$$
\Omega=\sum_{n=1}^{\infty} d t_{2 n} \theta^{2 n}+\sum_{n=1}^{\infty} d t_{2 n-1} \theta^{2 n-1}\left(1-\sum_{k=1}^{\infty} t_{2 k-1} \theta^{2 k-1}\right)
$$

Then a solution of the initial value problem for (2.21) $d U=\Omega U$ is unique in $\hat{\mathscr{E}}$.

Proof. Let $U$ and $V$ be two solutions with $\left.U\right|_{t=0}=\left.V\right|_{t=0}$. Let $W=U-V$. Using the same argument given above, we can conclude that $W$ does not depend on $t_{2 n}, n=1,2,3, \ldots$. Now, since

$$
\frac{\partial W}{\partial t_{2 n-1}}=\theta^{2 n-1}\left(1-\sum_{k=1}^{\infty} t_{2 k-1} \theta^{2 k-1}\right) W
$$

we have

$$
\left.\frac{\partial W}{\partial t_{2 n-1}}\right|_{t=0}=\left.\theta^{2 n-1} W\right|_{t=0}=0
$$

Thus $W$ does not depend on odd variables either. Thus Lemma 4.4 is established.

To establish the uniqueness of the super KP system (2.25), we have to show that every solution of $(2.25)$ gives rise to a solution of the universal equation (2.21). Namely, we need a super analogue of Theorem 4.1. For this purpose, we have to impose the following condition on the super differential algebra $(R, \theta)$;

Condition 4.5. There is a dual operator $x$ of the derivation $\theta^{2}: R \rightarrow R$ such that

(i) $\left[\theta^{2}, x\right]=1$.

(ii) $[R, x]=0$.

(iii) $[\theta, x]$ annihilates $R_{1}$, namely $a[\theta, x]=[\theta, x] a=0$ for all $a \in R_{1}$.

(iv) $[\theta, x]^{2}=0$.

The above condition will be satisfied if $R \cong A \otimes_{k} k \llbracket x, \xi \rrbracket$ and $\theta=\partial / \partial \xi+\xi \partial / \partial x$, where $A$ is a $k$-algebra consisting of even elements alone, $x$ is an even variable and $\xi$ is an odd variable with $\xi^{2}=0$. Condition 4.5 does not require that $x$ and $[\theta, x]$ are contained in $R$. Now we can establish the following;

Theorem 4.6. Let

$\omega=\sum_{n=1}^{\infty} d t_{2 n} P_{2 n}+\sum_{n=1}^{\infty} d t_{2 n-1} P_{2 n-1}+\sum_{n=1}^{\infty} \sum_{k=1}^{\infty} d t_{2 n-1} \cdot t_{2 k-1} P_{2 n+2 k-2}$ 
be a $\mathscr{D}$-valued 1 -form defined over a super differential algebra $(R, \theta)$ with Condition 4.5 satisfying that

(a) there is a positive constant $c$ such that ord $P_{n} \leqq \frac{n}{c}$ for all $n \geqq 1$, where $P_{2 n} \in \mathscr{D}_{0}$ and $P_{2 n-1} \in \mathscr{D}_{1}$, and

(b) $\omega$ is integrable, namely, $d \omega=\omega \wedge \omega$.

Then for every given element $Y(0) \in D_{0}$, there exists a unique solution $Y \in \hat{\mathscr{D}}_{0}$ of the linear total differential equation

$$
d Y=\omega Y
$$

with the initial value $\left.Y\right|_{t=0}=Y(0) \in D_{0}$.

Proof. Note that (4.13) is equivalent to the following systems;

$$
\begin{gathered}
\frac{\partial Y}{\partial t_{2 n}}=P_{2 n} \cdot Y \quad \text { for all } n=1,2,3, \ldots, \\
\frac{\partial Y}{\partial t_{2 n-1}}-\sum_{k=1}^{\infty} t_{2 k-1} \frac{\partial Y}{\partial t_{2 n+2 k-2}}=P_{2 n-1} \cdot Y \quad \text { for all } n=1,2,3, \ldots
\end{gathered}
$$

We will first solve the even system (4.14) consistently assuming that $t_{2 n-1}=0$ for all $n \geqq 1$, and then solve the odd part (4.15) consistently.

Since the super differential algebra $(R, \theta)$ satisfies Condition 4.5 , there is an operator $x$ with (i) (iv). Let us denote $[\theta, x]=\xi$ which is an odd operator on $R$. We want to use the same idea developed in the proof of Theorem 4.1 to show the solvability of (4.14). So we introduce the following notations. For every $n \geqq 0$, we define

$$
\begin{aligned}
\lambda^{2 n} & =x^{n} \\
\lambda^{2 n+1} & =\xi x^{n} \\
A \cdot[, \lambda]^{2 n} & =A \cdot[, x]^{n}=[[\ldots[[A, \underbrace{x], x], \ldots, x], x]}_{n \text {-times }} \\
A \cdot[, \lambda]^{2 n+1} & =\left[A \cdot[, x]^{n}, \xi\right] .
\end{aligned}
$$

The formulas we need are the following. Let $P_{2 n}=\sum_{j \geqq 0} a_{2 n, j} \theta^{j} \in \mathscr{D}_{0}$ be an even
super differential operator. Then we have

$$
\sum_{j \geqq l}\left[\begin{array}{l}
j \\
l l
\end{array}\right] a_{2 n, j} \theta^{j-l}=P_{2 n} \cdot \frac{[, \lambda]^{l}}{\left[\frac{l}{2}\right] !}
$$

and

$$
P_{2 n} \cdot \frac{\lambda^{j}}{\left[\frac{j}{2}\right] !}=\sum_{k=0}^{j} \frac{\lambda^{j-k}}{\left[\frac{j-k}{2}\right] !} P_{2 n} \cdot \frac{[, \lambda]^{k}}{\left[\frac{k}{2}\right] !}
$$

Using the above formulas we can show the following; 
Claim. The equation

is equivalent to

$$
\frac{\partial Y}{\partial t_{2 n}}=P_{2 n} \cdot Y \quad \text { for } Y=\sum_{l=0}^{\infty} y_{l} \theta^{l} \in \hat{\mathscr{D}}_{0}
$$

for all $l \geqq 0$.

$$
\frac{\partial}{\partial t_{2 n}}\left(\sum_{i=0}^{i} y_{l-i} \frac{\lambda^{i}}{\left[\frac{i}{2}\right] !}\right)=P_{2 n}\left(\sum_{i=0}^{l} y_{l-i} \frac{\lambda^{i}}{\left[\frac{i}{2}\right] !}\right)
$$

Since a super analogue of Lemma 4.2 can be easily established, (4.22) gives a unique formal solution $Y$ by the same argument we used in the proof of Theorem 4.1.

Proof of (4.20). We use a $\mathbf{Z}_{2}$-graded induction on $l$. If $l=0$, then (4.20) simply says $P_{2 n}=P_{2 n}$. So assume that (4.20) is true for an even number $l=2 m$. We want to show that it is also true for $l=2 m+1$ and $l=2 m+2$.

Now, by (4.19) we have

$$
\begin{aligned}
P_{2 n} \cdot \frac{[, \lambda]^{(2 m+1)}}{\left[\frac{2 m+1}{2}\right] !} & =\left[P_{2 n} \cdot \frac{[, \lambda]^{2 m}}{\left[\frac{2 m}{2}\right] !}, \xi\right]=\left[\sum_{j \geqq 2 m}\left[\begin{array}{c}
j \\
2 m
\end{array}\right] a_{2 n, j} \theta^{j-2 m}, \xi\right] \\
& =\sum_{j \geqq 2 m}\left[\begin{array}{c}
j \\
2 m
\end{array}\right] a_{2 n, j} \theta^{j-2 m} \cdot \xi-\sum_{j \geqq 2 m}\left[\begin{array}{c}
j \\
2 m
\end{array}\right] a_{2 n, j}(-1)^{j} \xi \theta^{j-2 m} \\
& =\sum_{j \geqq 2 m}\left[\begin{array}{c}
j \\
2 m
\end{array}\right] a_{2 n, j}\left[\begin{array}{c}
j-2 m \\
1
\end{array}\right](-1)^{j-1} \theta^{j-2 m-1} \\
& =\sum_{j \geqq 2 m} \tilde{j}\left[\begin{array}{c}
j \\
2 m
\end{array}\right] a_{2 n, j} \theta^{j-2 m-1}=\sum_{j \geqq 2 m}\left[\begin{array}{c}
j \\
2 m+1
\end{array}\right] a_{2 n, j} \theta^{j-2 m-1}
\end{aligned}
$$

by the definition of super binary coefficients. This establishes $l=2 m+1$ case.

For $l=2 m+2$, we have

$$
\begin{aligned}
P_{2 n} \cdot \frac{[, \lambda]^{2 m+2}}{\left[\frac{2 m+2}{2}\right] !}= & \frac{1}{m+1}\left[P_{2 n} \cdot \frac{[, \lambda]^{2 m}}{\left[\frac{2 m}{2}\right] !}, x\right] \\
= & \frac{1}{m+1} \sum_{j \geqq 2 m}\left[\begin{array}{c}
j \\
2 m
\end{array}\right] a_{2 n, j}\left[\theta^{j-2 m}, x\right] \\
= & \frac{1}{m+1} \sum_{j \geqq 2 m}\left[\begin{array}{c}
j \\
2 m
\end{array}\right] a_{2 n, j}\left[\begin{array}{c}
j-2 m \\
1
\end{array}\right] \xi \theta^{j-2 m-1} \\
& +\frac{1}{m+1} \sum_{j \geqq 2 m}\left[\begin{array}{c}
j \\
2 m
\end{array}\right] a_{2 m, j}\left[\begin{array}{c}
j-2 m \\
2
\end{array}\right] \theta^{j-2 m-2} \\
= & \frac{1}{m+1} \sum_{j \geqq 2 m} j\left[\begin{array}{c}
j \\
2 m
\end{array}\right] a_{2 n, j} \cdot \xi \theta^{j-2 m-1} \\
& +\sum_{j \geqq 2 m} \frac{\left[\begin{array}{c}
j \\
2 m
\end{array}\right]\left[\frac{j-2 m}{2}\right]}{\frac{2 m+2}{2}} a_{2 n, j} \theta^{j-2 m-2} .
\end{aligned}
$$


We are assuming $a_{2 n, j} \xi=0$ for odd $j$, thus the first term vanishes. (Remember that we are working on even parameters now and assuming $t_{2 n-1}=0$ for all $n$.) Since it is easy to see that

we can conclude that

$$
\left[\begin{array}{c}
j \\
2 m+2
\end{array}\right]=\frac{\left[\begin{array}{c}
j \\
2 m
\end{array}\right]\left[\frac{j-2 m}{2}\right]}{\frac{2 m+2}{2}},
$$

$$
P_{2 n} \cdot \frac{[, \lambda]^{2 m+2}}{\left[\frac{2 m+2}{2}\right] !}=\sum_{j \geqq 2 m}\left[\begin{array}{c}
j \\
2 m+2
\end{array}\right] a_{2 n, j} \theta^{j-2 m-2} .
$$

It completes the proof of (4.20).

Proof of (4.21). We also use a $\mathbf{Z}_{2}$-graded induction. When $j=0,(4.21)$ simply says $P_{2 n}=P_{2 n}$. So assume that it is true for some $j=2 \mathrm{~m}$. Then,

$$
\begin{aligned}
& P_{2 n} \cdot \frac{\lambda^{2 m+1}}{\left[\frac{2 m+1}{2}\right] !}=\left[P_{2 n} \cdot \frac{\lambda^{2 m}}{\left[\frac{2 m}{2}\right] !}, \xi\right]+\xi P_{2 n} \cdot \frac{\lambda^{2 m}}{\left[\frac{2 m}{2}\right] !} \\
& =\left[\sum_{k=0}^{2 m} \frac{\lambda^{2 m-k}}{\left[\frac{2 m-k}{2}\right] !} P_{2 n} \cdot \frac{[, \lambda]^{k}}{\left[\frac{k}{2}\right] !}, \xi\right]+\sum_{k=0}^{2 m} \frac{\xi \lambda^{2 m-k}}{\left[\frac{2 m-k}{2}\right] !} P_{2 n} \cdot \frac{[, \lambda]^{k}}{\left[\frac{k}{2}\right] !} \\
& =\sum_{l=0}^{m} \frac{\lambda^{2 m-2 l}}{\left[\frac{2 m-2 l}{2}\right] !} P_{2 n} \cdot \frac{[, \lambda]^{2 l+1}}{\left[\frac{2 l}{2}\right] !}+\sum_{l=0}^{m} \frac{\lambda^{2 m-2 l+1}}{\left[\frac{2 m-2 l}{2}\right] !} P_{n} \cdot \frac{[, \lambda]^{2 l}}{\left[\frac{2 l}{2}\right] !} .
\end{aligned}
$$

On the other hand, we have

$$
\begin{aligned}
& \sum_{k=0}^{2 m+1} \frac{\lambda^{2 m+1-k}}{\left[\frac{2 m+1-k}{2}\right] !} P_{2 n} \cdot \frac{[, \lambda]^{k}}{\left[\frac{k}{2}\right] !} \\
& \quad=\sum_{l=0}^{m} \frac{\lambda^{2 m+1-2 l}}{\left[\frac{2 m+1-2 l}{2}\right] !} P_{2 n} \frac{[, \lambda]^{2 l}}{\left[\frac{2 l}{2}\right] !}+\sum_{l=0}^{m} \frac{\lambda^{2 m-2 l}}{\left[\frac{2 m-2 l}{2}\right] !} P_{2 n} \cdot \frac{[, \lambda]^{2 l+1}}{\left[\frac{2 l+1}{2}\right] !} .
\end{aligned}
$$

Therefore, we obtain (4.21) for $j=2 m+1$. Next,

$$
\begin{aligned}
P_{2 n} \cdot & \frac{\lambda^{2 m+2}}{\left[\frac{2 m+2}{2}\right] !}=\frac{1}{m+1}\left[P_{2 n} \cdot \frac{\lambda^{2 m}}{\left[\frac{2 m}{2}\right] !}, x\right]+\frac{x}{m+1} P_{2 n} \cdot \frac{\lambda^{2 m}}{\left[\frac{2 m}{2}\right] !} \\
& =\frac{1}{m+1} \sum_{k=0}^{2 m} \frac{\lambda^{2 m-k}}{\left[\frac{2 m-k}{2}\right] !} P_{2 n} \cdot \frac{[, \lambda]^{k+2}}{\left[\frac{k}{2}\right] !}+\frac{1}{m+1} \sum_{k=0}^{2 m} \frac{\lambda^{2 m+2-k}}{\left[\frac{2 m-k}{2}\right] !} P_{2 n} \cdot \frac{[, \lambda]^{k}}{\left[\frac{k}{2}\right] !} \\
& =\frac{1}{m+1} \sum_{k=0}^{2 m+2} \frac{\lambda^{2 m+2-k}}{\left[\frac{2 m+2-k}{2}\right] !} P_{2 n} \cdot \frac{[, \lambda]^{k}}{\left[\frac{k}{2}\right] !}\left(\frac{\left[\frac{k}{2}\right] !}{\left[\frac{k-2}{2}\right] !}+\frac{\left[\frac{2 m+2-k}{2}\right] !}{\left[\frac{2 m-k}{2}\right] !}\right) .
\end{aligned}
$$


In the above summation, if $k$ is an odd number, then $\lambda^{2 m+2-k} P_{2 n} \cdot[, \lambda]^{k}=0$ since $\xi a_{2 n, j}=0$ for odd $j$. And if $k=2 l$, then

$$
\frac{\left[\frac{k}{2}\right] !}{\left[\frac{k-2}{2}\right] !}+\frac{\left[\frac{2 m+2-k}{2}\right]}{\left[\frac{2 m-k}{2}\right] !}=m+1 .
$$

Hence (4.23) reduces to

$$
P_{2 n} \cdot \frac{\lambda^{2 m+2}}{\left[\frac{2 m+2}{2}\right] !}=\sum_{k=0}^{2 m+2} \frac{\lambda^{2 m+2-k}}{\left[\frac{2 m+2-k}{2}\right] !} P_{2 n} \cdot \frac{[, \lambda]^{k}}{\left[\frac{k}{2}\right] !} .
$$

Thus we have obtained (4.21) for $j=2 m+2$. This completes the proof of (4.21).

Proof of (4.22). Since we have

$$
\begin{aligned}
0 & =\frac{\partial Y}{\partial t_{2 n}}-P_{2 n} \cdot Y=\sum_{l=0}^{\infty} \frac{\partial y_{l}}{\partial t_{2 n}}-\sum_{k \geqq 0} a_{2 n, k} \theta^{k} \sum_{m=0}^{\infty} y_{m} \theta^{m} \\
& =\sum_{l=0}^{\infty} \frac{\partial y_{l}}{\partial t_{2 n}}-\sum_{k \geqq 0} \sum_{m=0}^{\infty} \sum_{i=0}^{\infty}\left[\begin{array}{c}
k \\
i
\end{array}\right](-1)^{m(k-i)} a_{2 n, k} y_{m}^{[i]} \theta^{k+m-i} \\
& =\sum_{l=0}^{\infty}\left(\frac{\partial y_{l}}{\partial t_{2 n}}-\sum_{m=0}^{\infty} \sum_{k \geqq 0}\left[\begin{array}{c}
k \\
l-m
\end{array}\right](-1)^{m(l-m)} a_{2 n, k} y_{m}^{[k+m-l]}\right) \theta^{l},
\end{aligned}
$$

the equation is equivalent to

$$
\begin{aligned}
\frac{\partial y_{l}}{\partial t_{2 n}} & =\sum_{k \geqq 0} \sum_{m=0}^{l}\left[\begin{array}{l}
k \\
m
\end{array}\right](-1)^{m(l-m)} a_{2 n, k} y_{l-m}^{[k-m]} \\
& =\sum_{m=0}^{l}(-1)^{m(l-m)} \sum_{k \geqq m}\left[\begin{array}{l}
k \\
m
\end{array}\right] a_{2 n, k} \theta^{k-m}\left(y_{l-m}\right) \\
& =\sum_{m=0}^{l}(-1)^{m(l-m)} P_{2 n} \cdot \frac{[, \lambda]^{m}}{\left[\frac{m}{2}\right] !}\left(y_{l-m}\right) .
\end{aligned}
$$

Therefore,

$$
\begin{aligned}
& \frac{\partial}{\partial t_{2 n}}\left(\sum_{i=0}^{l} y_{l-1} \frac{\lambda^{i}}{\left[\frac{i}{2}\right] !}\right)=\sum_{i=0}^{l}(-1)^{i(l-1)} \frac{\lambda^{i}}{\left[\frac{i}{2}\right] !} \frac{\partial y_{l-i}}{\partial t_{2 n}} \\
& =\sum_{i=0}^{l} \sum_{m=0}^{l-i}(-1)^{i(l-i)}(-1)^{m(l-i-m)} \frac{\lambda^{i}}{\left[\frac{i}{2}\right] !} P_{2 n} \cdot \frac{[, \lambda]^{m}}{\left[\frac{m}{2}\right] !}\left(y_{l-m-i}\right)
\end{aligned}
$$




$$
\begin{aligned}
& =\sum_{j=0}^{l} \sum_{m=0}^{l-j}(-1)^{(j-m)(t-j+m)}(-1)^{m(l-j)} \frac{\lambda^{j-m}}{\left[\frac{j-m}{2}\right] !} P_{2 n} \cdot \frac{[, \lambda]^{m}}{\left[\frac{m}{2}\right] !}\left(y_{l-j}\right) \\
& =\sum_{j=0}^{l}(-1)^{j(l-j)} \sum_{m=0}^{l-j}(-1)^{m(j+1)} \frac{\lambda^{j-m}}{\left[\frac{j-m}{2}\right] !} P_{2 n} \cdot \frac{[, \lambda]^{m}}{\left[\frac{m}{2}\right] !}\left(y_{l-j}\right) \\
& =\sum_{j=0}^{l}(-1)^{j(l-j)} P_{2 n} \cdot \frac{\lambda^{j}}{\left[\frac{j}{2}\right] !}\left(y_{l-j}\right)=P_{2 n}\left(\sum_{j=0}^{l} y_{l-j} \frac{\lambda^{j}}{\left[\frac{j}{2}\right] !}\right),
\end{aligned}
$$

where we used that $\lambda^{j-m} P_{2 n} \cdot[, \lambda]^{m}=0$ if $j$ is even and $m$ is odd. This completes the proof of the claim.

The well-definedness of $Y \in \hat{\mathscr{D}}_{0}$ can be shown by a similar argument we used in the proof of Theorem 4.1, so it is omitted here.

Thus we have determined $t_{2 n}$ dependence of $Y$. Finally, let us sketch how to determine odd time dependence of $Y$. Since

$$
\begin{aligned}
0 & =\frac{\partial Y}{\partial t_{2 n-1}}-\sum_{k=1}^{\infty} t_{2 k-1} \frac{\partial Y}{\partial t_{2 n+2 k-2}}-P_{2 n-1} Y \\
& =\sum_{l=0}^{\infty}\left(\frac{\partial y_{l}}{\partial t_{2 n-1}}-\sum_{k=1}^{\infty} t_{2 k-1} \frac{\partial y_{l}}{\partial t_{2 n+2 k-2}}\right) \theta^{l}-\sum_{k \geqq 0} a_{2 n-1, k} \theta^{k} \sum_{j=0}^{\infty} y_{j} \theta^{j} \\
& =\sum_{i=0}^{\infty}\left(\frac{\partial y_{l}}{\partial t_{2 n-1}}-\sum_{k=1}^{\infty} t_{2 k-1} \frac{\partial y_{l}}{\partial t_{2 n+2 k-2}}-\sum_{k \geqq 0} \sum_{j=0}^{\infty}\left[\begin{array}{c}
k \\
k+j-l
\end{array}\right] a_{2 n-1, k} y_{j}^{[k+j-l]}\right) \theta^{l},
\end{aligned}
$$

we have

$$
\frac{\partial y_{l}}{\partial t_{2 n-1}}-\sum_{k=1}^{\infty} t_{2 k-1} \frac{\partial y_{l}}{\partial t_{2 n+2 k-2}}=\sum_{j=0}^{l} \sum_{k \geq 0}\left[\begin{array}{l}
k \\
j
\end{array}\right] a_{2 n-1, k} y_{l-j}^{[k-j]} .
$$

For individual $t_{2 n-1}$ dependence, we can expand $y_{l}=y_{l, 0}+t_{2 n-1} y_{l, 1}$ and $a_{2 n-1, k}=a_{2 n-1, k, 0}+t_{2 n-1} a_{2 n-1, k, 1}$. Therefore, letting $t_{2 n-1}=0$, we obtain

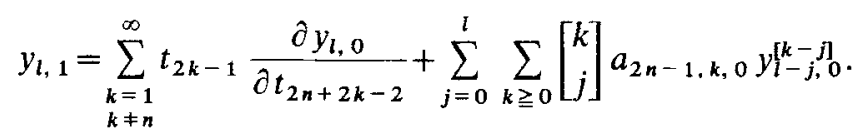

This means that $\left.y_{t}\right|_{t_{2 n-1}=0}$ determines $y_{l}$. Namely, $\left.Y\right|_{t_{\text {odd }}=0}$ determines $Y$ completely. Compatibility of $t$-dependence follows from the integrability $d \omega=\omega \wedge \omega$. Since ord $P_{2 n-1} \leqq(2 n-1) / c,(4.24)$ gives a lower bound for $\mathrm{val}_{t} y_{l, 1}$. Thus the well-definedness $Y \in \hat{\mathscr{D}}_{0}$ follows. This completes the proof of Theorem 4.6. 


\section{Examples}

In this section we give some of the explicit forms of differential equations obtained from the generalized KP system and the super KP system. From now on, we use the following convention: for a function $f$, we denote $\partial f / \partial x=f^{\prime}$ and $\partial f / \partial t_{n}=f_{, n}$.

Let $A$ be an associative $k$-algebra and let $R=A \otimes_{k} k \llbracket x \rrbracket$. Then the generalized $\mathrm{KP}$ system (1.19) produces the following equations;

$$
\begin{aligned}
& s_{1,2}=s_{1}^{\prime \prime}-2 s_{1}^{\prime} s_{1}+2 s_{2}^{\prime} \\
& s_{2,2}=s_{2}^{\prime \prime}-2 s_{1}^{\prime} s_{2}+2 s_{3}^{\prime} \\
& s_{1,3}=s_{1}^{\prime \prime \prime}-3 s_{1}^{\prime \prime} s_{1}-3\left(s_{1}^{\prime}\right)^{2}+3 s_{1}^{\prime} s_{1}^{2}+3 s_{2}^{\prime \prime}-3 s_{2}^{\prime} s_{1}-3 s_{1}^{\prime} s_{2}+3 s_{3}^{\prime} .
\end{aligned}
$$

Eliminating $s_{2}$ and $s_{3}$ from the above equations, we obtain the non-commutative $K P$ equation

$$
s_{1,3}^{\prime}-(3 / 4) s_{1,22}-(3 / 2)\left[s_{1}^{\prime}, s_{1,2}\right]-(1 / 4) s_{1}^{\prime \prime \prime}+(3 / 2)\left[\left(s_{1}^{\prime}\right)^{2}\right]^{\prime}=0 .
$$

When $A$ is commutative, then (5.1) reduces to the original KP equation on $u_{2}=-s_{1}^{\prime}$;

$$
4 u_{2,3}^{\prime}-3 u_{2,22}-u_{2}^{\prime \prime \prime}-12\left(u_{2} u_{2}^{\prime}\right)^{\prime}=0 .
$$

Now let's consider the super KP system. For a super differential algebra, we take $R=\mathbb{C}[x, \xi]$ and $\theta=\partial / \partial \xi+\xi \partial / \partial x$, where $x$ is an even variable and $\xi$ is an odd variable satisfying $\xi^{2}=0$. Since $\theta^{2}=\partial / \partial x$, the derivation map $\theta^{2}: R$ $\rightarrow R$ is surjective. Let

$$
L=\theta+u_{1}+u_{2} \theta^{-1}+u_{3} \theta^{-2}+\ldots
$$

be a monic first order super micro-differential operator of homogeneous $\mathbb{Z}_{2}$-degree 1 (i.e. $\tilde{u}_{n}=\tilde{n} \in \mathbb{Z}_{2}$ ). We assume the consistency condition

$$
u_{1}^{[1]}+2 u_{2}=0
$$

from now on. Then by Proposition 2.2 there is an $S \in G_{0}$ such that $L=S \theta S^{-1}$. Therefore, the Lax equation (2.27) is uniquely solvable for this $L$. To compute actual forms of (super) differential equations, we need the following:

$$
\begin{aligned}
L^{2}= & \theta^{2}+u_{2}^{[1]} \theta^{-1}+\left(2 u_{4}+u_{3}^{[1]}-u_{2}^{2}\right) \theta^{-2}+\left(u_{4}^{[1]}-u_{2} u_{2}^{[1]}\right) \theta^{-3}+\ldots, \\
L^{3}= & \theta^{3}+u_{1} \theta^{2}+u_{2} \theta+\left(u_{3}-u_{2}^{[1]}\right)+\left(3 u_{4}+u_{3}^{[1]}+u_{2}^{[2]}-u_{2}^{2}+u_{1} u_{2}^{[1]}\right) \theta^{-1} \ldots, \\
L^{4}= & \theta^{4}+2 u_{2}^{[1]} \theta+2\left(2 u_{4}+u_{3}^{[1]}-u_{2}^{2}\right)+\left(2 u_{4}^{[1]}+u_{2}^{[3]}-2 u_{2} u_{2}^{[1]}\right) \theta^{-1} \ldots, \\
L^{6}= & \theta^{6}+3 u_{2}^{[1]} \theta^{3}+3\left(2 u_{4}+u_{3}^{[1]}-u_{2}^{2}\right) \theta^{2}+3\left(u_{4}^{[1]}+u_{2}^{[3]}-u_{2} u_{2}^{[1]}\right) \theta \\
& +3\left(2 u_{6}+u_{5}^{[1]}+2 u_{4}^{[2]}+u_{3}^{[3]}+u_{2} u_{3}^{[1]}-2 u_{2}^{[1]} u_{3}-u_{2} u_{2}^{[2]}\right) \\
& +\left(3 u_{6}^{[1]}+3 u_{4}^{[3]}+3 u_{2}^{[1]} u_{4}+3 u_{2} u_{4}^{[1]}+3 u_{2} u_{3}^{[2]}+6 u_{2}^{[1]} u_{3}^{[1]}\right. \\
& \left.-3 u_{2}^{[2]} u_{3}+u_{2}^{[5]}-6 u_{2}^{2} u_{2}^{[1]}\right) \theta^{-1} \ldots
\end{aligned}
$$


Substituting the above expression in (2.27), one obtains

$$
\begin{aligned}
u_{2,1}= & -u_{2}^{[1]}+\sum_{n=1}^{\infty} t_{2 n-1} u_{2,2 n}, \\
u_{3,1}= & -2 u_{4}-u_{3}^{[1]}+\sum_{n=1}^{\infty} t_{2 n-1} u_{3,2 n}, \\
u_{2,3}= & -3 u_{4}^{[1]}-u_{3}^{[2]}-u_{2}^{[3]}+4 u_{2} u_{2}^{[1]}+u_{1} u_{2}^{[2]}+\sum_{n=1}^{\infty} t_{2 n-1} u_{2,2 n+2}, \\
u_{2,4}= & 2 u_{4}^{[2]}+u_{2}^{[4]}-2 u_{2} u_{2}^{[2]}, \\
u_{4,4}= & 2 u_{6}^{[2]}+u_{4}^{[4]}+4 u_{2} u_{4}^{[2]}-2 u_{2}^{[1]} u_{4}^{[1]}-2 u_{2}^{[2]} u_{4}+2 u_{2} u_{3}^{[3]} \\
& -2 u_{2}^{[3]} u_{3}+2 u_{2} u_{2}^{[4]}-4 u_{2}^{2} u_{2}^{[2]}, \\
u_{2,6}= & 3 u_{6}^{[2]}+3 u_{4}^{[4]}+3 u_{2} u_{4}^{[2]}+3 u_{2}^{[2]} u_{4}+3 u_{2} u_{3}^{[3]}-3 u_{2}^{[1]} u_{3}^{[2]} \\
& +3 u_{2}^{[2]} u_{3}^{[1]}-3 u_{2}^{[3]} u_{3}+u_{2}^{[6]}-6 u_{2}^{2} u_{2}^{[2]} .
\end{aligned}
$$

For the purpose of elimination, we introduce a new variable $v_{0}$ such that

$$
v_{0}^{[2]}=u_{2} .
$$

Then we obtain a closed system of equations for $v_{0}$ and $u_{3}$ :

$$
\begin{gathered}
2 v_{0,3}^{[3]}+3 u_{3,1}^{[2]}-\sum_{n=1}^{\infty} t_{2 n-1}\left(2 v_{0,2 n+2}^{[3]}+3 u_{3,2 n}^{[2]}\right) \\
=2 v_{0}^{[6]}-4 v_{0}^{[1]} v_{0}^{[5]}-4 v_{0}^{[2]} v_{0}^{[4]}-u_{3}^{[3]}, \\
4 v_{0,6}^{[2]}-3 v_{0,44}-12 v_{0}^{[4]} v_{0,4}-6 v_{0}^{[3]} v_{0,4}^{[1]} \\
=v_{0}^{[8]}-6 v_{0}^{[3]} v_{0}^{[5]}-6\left(v_{0}^{[4]}\right)^{2}+12 v_{0}^{[4]} u_{3}^{[1]}-12 v_{0}^{[3]} u_{3}^{[2]} .
\end{gathered}
$$

To eliminate further $u_{3}$ from (5.4) and (5.5) above, let us expand $v_{0}$ and $u_{3}$ in the following form:

$$
\left\{\begin{array}{l}
v_{o}=f_{0}+t_{1} \xi f_{2}+t_{3} \xi f_{4}+t_{1} t_{3} h_{4}+\ldots \\
u_{3}=\xi g_{4}+t_{1} q_{4}+t_{3} g_{6}+t_{1} t_{3} \xi g_{8}+\ldots
\end{array}\right.
$$

where $f_{0}, f_{2}, f_{4}, h_{4}, q_{4}, g_{4}, g_{6}$ and $g_{8}$ are all functions in $\mathbb{C} \llbracket x, t_{4}, t_{6}, \ldots \rrbracket$. Note that we can identify $t_{2}$-dependence with $x$-dependence because

$$
\partial L / \partial t_{2}=\left[\theta^{2}, L\right]=[\partial / \partial x, L]=\partial L / \partial x .
$$

We write $f^{[2]}=f^{\prime}$ etc. from now on. Then Eq. (5.4) gives the following:

$$
\begin{aligned}
& f_{2}=-f_{0}^{\prime}, \\
& h_{4}=f_{4}+f_{0,4}, \\
& g_{4}=-(3 / 2) f_{0,4}+(1 / 2) f_{0}^{\prime \prime}-(1 / 2)\left(f_{0}^{\prime}\right)^{2}-f_{4}, \\
& q_{4}=(1 / 2) f_{0,4}+(1 / 2) f_{0}^{\prime \prime}-(1 / 2)\left(f_{0}^{\prime}\right)^{2}+f_{4}, \\
& g_{6}^{\prime}=f_{0,6}^{\prime}+(3 / 2) f_{4,4}-(1 / 2) f_{4}^{\prime \prime}-f_{0}^{\prime} f_{4}^{\prime}+2 f_{0}^{\prime \prime} f_{4}, \\
& g_{8}=f_{0,6}^{\prime}-(1 / 2) f_{4,4}-(3 / 2) f_{0,44}+(1 / 2) f_{0,4}^{\prime \prime}-f_{0}^{\prime} f_{0,4}^{\prime}+(1 / 2) f_{4}^{\prime \prime}-3 f_{0}^{\prime} f_{4}^{\prime}+2 f_{0}^{\prime \prime} f_{4} .
\end{aligned}
$$


Substituting the above expressions into Eq. (5.5) together with (5.6), we obtain the following system of closed non-supersymmetric (i.e. in the usual sense of) differential equations;

$$
\begin{gathered}
f_{0}^{\prime \prime \prime} f_{4}^{\prime}-f_{0}^{\prime \prime} f_{4}^{\prime \prime}+f_{0}^{\prime \prime} f_{4,4}-f_{0,4}^{\prime} f_{4}^{\prime}=0, \\
4 f_{0,6}^{\prime}-3 f_{0,44}+6 f_{0}^{\prime \prime} f_{0,4}-f_{0}^{\prime \prime \prime \prime}+6\left(f_{0}^{\prime}\right)^{2} f_{0}^{\prime \prime}+12 f_{0}^{\prime \prime} f_{4}=0 .
\end{gathered}
$$

Equation (5.8) has an obvious solution

$$
f_{4}=\lambda^{2} f_{0}^{\prime}
$$

where $\lambda \in \mathbb{C}$ is a spectral parameter introduced to keep consistency of the weighted homogeneous degree of variables. (The degree we are using is the following: $\operatorname{deg} x=-2, \operatorname{deg} \xi=-1, \operatorname{deg} t_{n}=-n, \operatorname{deg} \lambda=1$ and $\operatorname{deg} f_{n}=n$.) Thus one can eliminate $f_{4}$ in Eq. (5.9) and finally obtains the following new nonlinear integrable partial differential equation;

$$
4 f_{0,6}^{\prime}-3 f_{0,44}+6 f_{0}^{\prime \prime} f_{0,4}-f_{0}^{\prime \prime \prime \prime}+6\left(f_{0}^{\prime}\right)^{2} f_{0}^{\prime \prime}+12 \lambda^{2} f_{0}^{\prime} f_{0}^{\prime \prime}=0 .
$$

The original unknown function $u_{2}$ of the super KP system is given by

$$
u_{2}=f_{0}^{\prime}-t_{1} \xi f_{0}^{\prime \prime}+t_{3} \xi \lambda^{2} f_{0}^{\prime \prime}+t_{1} t_{3}\left(\lambda^{2} f_{0}^{\prime \prime}+f_{0,4}^{\prime}\right)+\ldots .
$$

In (5.7) we have expressed everything in terms of $f_{0}$ and $f_{4}$. In connection with the argument of Sect. 4 , we can observe the following fact here; since $f_{4}$ is uniquely expressible in terms of $f_{0}$ and $g_{4}$ by (5.7), $v_{0}$ and $u_{3}$ are completely determined by $\left.v_{0}\right|_{t_{\text {odd }}=0}=f_{0}$ and $\left.u_{3}\right|_{t_{\text {odd }}=0}=\xi g_{4}$. In other words, once we determine all even time dependence under the condition of $t_{\text {odd }}=0$, then terms with odd variables are uniquely determined automatically by the super KP system.

Let us now solve Eq. (5.10). We introduce propergation speeds $a$ and $b$ and set

$$
f_{0}=\phi\left(\lambda^{2} x+a \lambda^{4} t_{4}+b \lambda^{6} t_{6}\right) .
$$

Then Eq. (5.10) reduces to an ordinary differential equation of $\phi=\phi(z)$;

$$
\phi_{z z z z}=6 \phi_{z}^{2} \phi_{z z}+12(a / 2+1) \phi_{z} \phi_{z z}+4\left(b-(3 / 4) a^{2}\right) \phi_{z z} .
$$

We can integrate this equation to obtain

$$
\phi_{z z z}=2 \phi_{z}^{3}+3(a+2) \phi_{z}^{2}+\left(4 b-3 a^{2}\right) \phi_{z}+(1 / 2) c_{1}
$$

with a constant of integration. Taking one more integration of (5.14) after multiplying $\phi_{z z}$ to the both hand sides, we have

$$
\psi_{z}^{2}=\psi^{4}+2(a+2) \psi^{3}+\left(4 b-3 a^{2}\right) \psi^{2}+c_{1} \psi+c_{2},
$$

where we have introduced $\psi=\phi_{z}$ and another constant of integration. The general solution of Eq. (5.15) can be expressed in terms of the Jacobi elliptic functions [14]. When $a=b=0$, the original function $u_{2}$ does not depend on $t_{4}$ and $t_{6}$. 
A solution $\psi=4 /\left(4 z^{2}-1\right)$ of $(5.15)$ gives a rational solution

$$
u_{2}=\frac{\lambda^{2}}{\lambda^{4} x^{2}-(1 / 4)}+t_{1} \xi \frac{2 \lambda^{6} x}{\left(\lambda^{4} x^{2}-(1 / 4)\right)^{2}}-t_{3} \xi \frac{2 \lambda^{8} x}{\left(\lambda^{4} x^{2}-(1 / 4)\right)^{2}}+\ldots
$$

in terms of $u_{2}$. The corresponding solution of Eq. (5.10) is

$$
f_{0}=\log \left[\left(2 \lambda^{2} x-1\right) /\left(2 \lambda^{2} x+1\right)\right] .
$$

If we take $a=-2$ and $b=5 / 2$, then $\psi=\cosh (z) / \sinh (z)$ is a solution of (5.15). It gives a soliton-like solution

$$
u_{2}=\lambda^{2} \frac{\cosh \left(\lambda^{2} x-2 \lambda^{4} t_{4}+(5 / 2) \lambda^{6} t_{6}\right)}{\sinh \left(\lambda^{2} x-2 \lambda^{4} t_{4}+(5 / 2) \lambda^{6} t_{6}\right)}+[\text { terms with odd variables }] .
$$

The first term of the above solution, which is equal to $f_{0}^{\prime}$, is not exactly a soliton because of its boundary behavior, however, the $x$-derivative $f_{0}^{\prime \prime}$ behaves very much like a soliton. Although it has a singularity, if we shift the $x$-axis little bit to the imaginary direction, then we obtain a one-soliton solution. From this point of view, we want to call Eqs. (5.8) to (5.10) soliton equations.

Finally, if we set $a=-2$ and $b=7-k^{2} / 2$, then Eq. (5.15) has a solution $\psi=c n(z) / \sin (z)$, where $\operatorname{sn}(z)$ and $c n(z)$ are the Jacobi elliptic functions of modulus $k$. This gives a quasi-periodic solution

$$
u_{2}=\lambda^{2} \frac{c n\left(\lambda^{2} x-2 \lambda^{4} t_{4}+\left(7-k^{2} / 2\right) \lambda^{6} t_{6}\right)}{\operatorname{sn}\left(\lambda^{2} x-2 \lambda^{4} t_{4}+\left(7-k^{2} / 2\right) \lambda^{6} t_{6}\right)}+[\text { terms with odd variables }]
$$

in terms of the original function $u_{2}$. Thus Eqs. (5.8) to (5.10) enjoy the common properties which usual soliton equations such as $\mathrm{KdV}$ equation, KP equation and others possess. We can also observe the formal resemblance among (5.2) and (5.10). For example, their linear terms are identical. Moreover, when the parameter $\lambda$ is set to be 0 , then (5.10) reduces to the first modified KP equation, whose integrability has been well understood. For example, it has a bilinear expression of Hirota. (Hirota has shown recently that (5.10) can be recovered from the first modified KP equation by a simple coordinate transformation.)

However, it should be noted that the new equations (5.8) and (5.9) cannot be obtained from the KP equation (5.2). Of course the super KP system involves the KP system. Therefore, we can derive all the hierarchy of the KP equations from the super KP system. But the latter has much more equations which have no counterpart in the usual KP framework even in the even variables. Our coupled system of (5.8) and (5.9) are examples of such equations. In the usual $\mathrm{KP}$ framework, the unknown function $u_{2}$ (or $f_{0}$ ) is equal to 0. Therefore, Eqs. (5.8) to (5.10) emerge only if we generalize the framework of the KP theory into the category of supersymmetry.

The KP equation is a unification of the $\mathrm{KdV}$ equation and the Boussinesq equation. In the notations of Introduction, if we set $u_{y}=0$ in (2), integrate the whole expression by $x$ and set the constant of integration to be 0 , then we recover the $\mathrm{KdV}$ equation $u_{t}=(1 / 4) u_{x x x}+3 u u_{x}$. On the other hand, if we set $u_{t}=0$ in (2), then it becomes the Boussinesq equation. In a similar sense, we 
have shown that the super KP system is a natural unification of the KP system and the first modified KP system. Namely, if we take the reduction of the super KP system modulo odd variables, then we recover the KP system. On the other hand, if we eliminate odd variables in the super KP system, then we can obtain the first modified KP system.

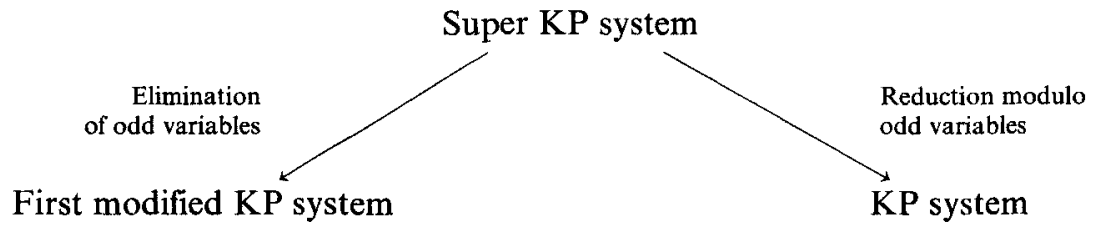

Thus we can conclude that the extension into the category of supersymmetry is not a trivial formalism generalization, but an essential step. As we have observed, a supersymmetric extension of the KP system produces new nonlinear differential equations. In this paper we have established the complete integrability of those new equations.

\section{References}

1. Atiyah, M.F.: Commentary on the article of Manin. In: Arbeitstagung Bonn 1984. Lect. Notes in Math., vol. 1111, pp. 103-109. Berlin Heidelberg New York Tokyo: Springer 1985

2. Connes, A.: Introduction to non commutative differential geometry. ibid. 1111, 3-16 (1985)

3. Date, E., Jimbo, M., Kashiwara, M., Miwa, T.: Transformation groups for soliton equations. In: Proc. Symp. on Non-linear Integrable Systems, RIMS, Kyoto Univ., ed. by Jimbo and Miwa, World Scientific, pp. 39-120 (1983)

4. Lax, P.D.: Integrals of nonlinear equations of evolution and solitary waves. Commun. Pure Appl. Math. 21, 467-490 (1968)

5. Manin, Yu.I.: New dimensions in geometry. In: Arbeitstagung Bonn 1984. Lect. Notes in Math., vol. 1111, pp. 59-101. Berlin Heidelberg New York Tokyo: Springer 1985

6. Manin, Yu.I., Radul, A.O.: A supersymmetric extension of the Kadomtsev-Petviashvili hierarchy. Commun. Math. Phys. 98, 65-77 (1985)

7. Mulase, M.: Algebraic geometry of soliton equations. Proc. Japan Acad., Ser. A 59, 285-288 (1983)

8. Mulase, M.: Cohomological structure in soliton equations and jacobian varieties. J. Differ. Geom. $19,403-430$ (1984)

9. Mulase, M.: Complete integrability of the Kadomtsev-Petviashvili equation. Adv. Math. 54, 57-66 (1984)

10. Pressley, A., Segal, G.B.: Loop groups. Oxford Univ. Press (1986)

11. Sato, M., Sato, Y.: Soliton equations as dynamical systems on infinite dimensional Grassmann manifold. In: Nonlinear PDE in Applied Science. U.S.-Japan Seminar, Tokyo, 1982. Lect. Notes in Num. Appl. Anal. vol. 5, pp. 259-271. Tokyo: Kinokuniya 1982

12. Segal, G.B., Wilson, G.: Loop groups and equations of KdV type. Publ. Math. Inst. Hautes Etud. Sci. 61, 5-65 (1985)

13. Ueno, K., Yamada, H.: A supersymmetric extension of nonlinear integrable systems. In: Hietarinta, J., Westerholm, J. (eds.). Topological and geometrical methods in field theory. World Scientific, pp. 59-72 (1986), see also: Supersymmetric extension of the Kadomtsev-Petviashvili hierarchy and the universal super Grassmann manifold. Adv. Stud. Pure Math., Kinokuniya (to appear 1987)

14. Whittaker, E.T., Watson, G.N.: A course of modern analysis. Cambridge University Press 1927

15. Witten, E.: Physics and geometry (To appear in Proc. of the ICM-86, Berkeley) 